\title{
ON THE VALIDITY OR FAILURE OF GAP RIGIDITY FOR CERTAIN PAIRS OF BOUNDED SYMMETRIC DOMAINS*
}

\author{
PHILIPPE EYSSIDIEUX ${ }^{\dagger}$ AND NGAIMING MOK ${ }^{\ddagger}$
}

In memory of Professor Armand Borel

Let $\Omega$ be a bounded symmetric domain equipped with a canonical Kähler metric. We are interested to characterize holomorphic geodesic cycles (i.e., compact complex geodesic submanifolds) $S \subset X$ on Hermitian locally symmetric manifolds $X$ uniformized by $\Omega$, i.e., $X=\Omega / \Gamma$, where $\Gamma \subset \operatorname{Aut}(\Omega)$ is any torsion-free discrete group of automorphisms, in terms of differential-geometric or algebro-geometric conditions. In Eyssidieux-Mok [EysMok1995] we studied almost geodesic complex submanifolds and formulated the gap phenomenon. Up to equivalence under Aut $(\Omega)$ there are only a finite number of totally geodesic complex submanifolds $D \subset \Omega$ which are themselves biholomorpic to bounded symmetric domains. Let $\epsilon>0$. We say that $S \subset X$ is $\epsilon$-geodesic if and only if the norm of the second fundamental form of $S \subset X$ is uniformly bounded by $\epsilon$. Fixing $\Omega$ but letting $\Gamma \subset \Omega$ be arbitrary we showed that when $\epsilon$ is sufficiently small, an $\epsilon$-geodesic compact complex submanifold $S \subset X$ must locally resemble one and only one embedding of the models $(\Omega, D ; i), i: D \hookrightarrow \Omega$. We say that gap rigidity holds for the pair $(\Omega, D ; i)$ if for $\epsilon$ sufficiently small, any $\epsilon$-geodesic compact complex submanifold $S \subset X$ modeled on $(\Omega, D ; \imath)$ is necessarily totally geodesic for any $X$ uniformized by $\Omega$. When $i: D \hookrightarrow \Omega$ is understood, we will sometimes just write $(\Omega, D)$.

There is a strong form of the gap phenomenon, where one can characterize certain holomorphic geodesic cycles in algebro-geometric terms, in terms of the genericity of their tangent spaces. Write $\Omega=G / K$, where $G=\operatorname{Aut}(\Omega)$ and $K \subset G$ is the isotropy subgroup at a reference point $o \in \Omega$. We will say that gap rigidity holds for $(\Omega, D)$ in the Zariski topology if there exists a $K$-invariant Zariski-open subset $\mathcal{O}_{o}$ in the Grassmannian $\operatorname{Gr}\left(\operatorname{dim}(D), T_{o}(\Omega)\right)$ of $\operatorname{dim}(D)$-planes in $T_{o}(\Omega)$ such that $\left[T_{o}(D)\right] \in \mathcal{O}_{o}$, and such that, for any complex manifold $X$ uniformized by $\Omega$, any compact complex submanifold $S \subset X$ of dimension equal to $\operatorname{dim}(D)$ must be totally geodesic, whenever every tangent plane $\left[T_{x}(S)\right]$ of $S$ lifts to an element of $\mathcal{O}_{o}$. Obviously if gap rigidity holds for $(\Omega, D)$ in the Zariski topology, it holds for $(\Omega, D)$ in the differential-geometric sense as explained. To make a distinction we will sometimes refer to the latter as gap rigidity in the complex topology.

The simplest example of gap rigidity in the Zariski topology is the case of a product domain $\left(D^{k}, D ; \delta\right)$, where $\delta$ always refers to the diagonal embedding, given by $\delta(z)=(z, \cdots, z)$, as given in [EysMok1995]. The observation there resulted from the uniqueness of Kähler-Einstein metrics on a compact complex manifold with ample canonical line bundle. Eyssidieux [Eys1997][Eys1999] considered, in the context of variations of Hodge structures, the question of characterizing certain holomorphic geodesic cycles by Chern number inequalities, establishing as a by-product gap rigidity

\footnotetext{
${ }^{*}$ Received March 16, 2004; accepted for publication May 14, 2004.

${ }^{\dagger}$ CNRS - Laboratoire Emile Picard, Université Paul Sabatier, Toulouse, France (eyssi@picard. ups-tlse.fr).

$\ddagger$ Department of Mathematics, The University of Hong Kong, Hong Kong, China (nmok@ hkucc.hku.hk). Research supported by a CERG grant of the Research Grants Council of HongKong.
} 
in the Zariski topology for many pairs of $(\Omega, D)$ by means of Gauss-Manin complexes. His methods apply to the case of period domains arising from Hodge theory. In Mok [Mok2002] using intersection theory on the projectivized tangent bundle we determined the set of all pairs $(\Omega, D)$ with $\Omega$ irreducible and $\operatorname{dim}(D)=1$ for which gap rigidity holds in the Zariski topology. They are precisely those $\Omega$ of rank $r>1$ such that the highest characteristic subvariety (cf. [Mok2002],§1) $\mathcal{S}_{o} \subset \mathbb{P}\left(T_{o}(\Omega)\right.$ ) is a hypersurface in $\mathbb{P}\left(T_{o}(S)\right)$. At the same time, exploiting the existence and uniqueness of Kähler-Einstein metrics on projective manifolds with ample canonical line bundle, G-structures, and Hermitian metric rigidity, we proved that for $\Omega=D_{n}^{I V}$ the $n$-dimensional bounded symmetric domain of type IV (dual to the hyperquadric) and of dimension $\geq 3$, gap rigidity holds in the Zariski topology for $\left(D_{n}^{I V}, D_{k}^{I V}, i\right)$, where $k \geq 2$ and $i: D_{k}^{I V} \hookrightarrow D_{n}^{I V}$ is the standard embedding.

The purpose of the article is two-fold. First of all, we will show that in general gap rigidity already fails in the complex topology. More precisely, we show that gap rigidity fails for $\left(\Delta^{2}, \Delta \times\{0\}\right)$ by constructing a sequence of ramified coverings $f_{i}: S_{i} \rightarrow T_{i}$ between hyperbolic compact Riemann surfaces such that, with respect to norms defined by the Poincaré metrics, $\sup \left\|d f_{i}\right\| \rightarrow 0$ as $i \rightarrow \infty$. Since any bounded symmetric domain of rank $\geq 2$ contains a totally geodesic bidisk, this implies that gap rigidity fails in general on any bounded symmetric domain of rank $\geq 2$. Our counterexamples make it all the more interesting to find sufficient conditions for pairs $(\Omega, D)$ for which gap rigidity holds. This will be addressed in the second part of the article, where for $\Omega$ irreducible, we generalize the results for holomorphic curves in [Mok2002] to give a sufficient condition for gap rigidity to hold for $(\Omega, D)$ in the Zariski topology. In notations of the preceding paragraphs, we show that it is sufficient to take $\mathcal{O}_{o} \subset T_{o}(\Omega)$ to be such that the excluded subvariety $\mathcal{Z}_{o}=\operatorname{Gr}\left(\operatorname{dim}(D), T_{o}(\Omega)\right)-\mathcal{O}_{o}$ is a hypersurface. In the terminology of geometric invariant theory we say that $\left[T_{o}(D)\right] \in$ $\operatorname{Gr}\left(\operatorname{dim}(D), T_{o}(\Omega)\right)$ is a semistable point. For the case of bounded symmetric domains, this criterion applies to the case of 1-hyperrigid domains $D \subset \Omega$ of [Eys1997][Eys1999] and to the examples of [Mok2002] for holomorphic curves and from quadric structures, giving a unifying conceptual explanation and a more general framework for the validity of the phenomenon. In the case where $D$ is irreducible, we will show that the class of GIT-semistable embeddings of irreducible bounded symmetric domains coincides with the class of $\left(H_{2}\right)$-embeddings, see [Sat1980]. In [Sat1965], I. Satake classified $\left(H_{2}\right)$-embeddings into classical domains and the full classification has been obtained by S. Ihara [Iha1967] as a step towards his classification of all embeddings of bounded symmetric domains. Since the abstract classification of GIT-semistable embeddings does not give an explicit invariant hypersurface in each case, we will also give a detailed exposition of some examples.

\section{Failure of gap rigidity on the bidisk.}

1.1. Statement of main result and basic reductions. For a product domain $D^{k}$ denote by $\delta: D \rightarrow D^{k}$ the diagonal embedding given by $\delta(z)=(z, \cdots, z)$. By Eyssidieux-Mok [EysMok1995], for any bounded symmetric domain $D$, gap rigidity in the Zariski topology holds for $\left(D^{k}, D ; \delta\right)$. It turns out that the analogue is in general not valid when $\delta$ is replaced by another holomorphic totally geodesic embedding.

The main result of this section is to give a counterexample to the gap phenomenon as formulated in Eyssidieux-Mok [EysMok1995] in this context.

TheOREM 1. Gap rigidity fails on the bidisk for $\left.\left(\Delta^{2}, \Delta \times\{0\}\right)\right)$.

Since any bounded symmetric domain $\Omega$ of rank $\geq 2$ contains a totally geodesic 
bidisk, Theorem 1 implies that gap rigidity fails in general whenever $\Omega$ is of rank $\geq 2$. We are going to prove Theorem 1 by constructing compact holomorphic curves on products of compact Riemann surfaces of genus $\geq 2$ which are graphs of holomorphic maps. The requirement that they become $\epsilon$-geodesic and modeled on $\left(\Delta^{2}, \Delta \times\{0\}\right)$ ) then translates to a uniform bound on the differential of the holomorphic map in terms of Poincaré metrics, as follows.

THEOREM 2. There exist sequences of compact Riemann surfaces $S_{i}$ and $T_{i}$ of genus $\geq 2$, together with a sequence of branched double covers $f_{i}: S_{i} \rightarrow T_{i}$, such that, writing $d s_{C}^{2}$ for the Poincaré metric of Gaussian curvature -2 on a compact Riemann surface $C$ of genus $\geq 2$, and putting $\mu_{i}:=\sup \left\{\frac{f_{i}^{*} d s_{T_{i}}^{2}(x)}{d s_{S_{i}}^{2}(x)}: x \in S_{i}\right\}$, we have $\lim _{i \rightarrow \infty} \mu_{i}=0$.

We now prove that Theorem 2 implies Theorem 1.

Proof. Let $G_{i} \subset S_{i} \times T_{i}$ be the graph of $f_{i}$. Let $F_{i}: \Delta \rightarrow \Delta$ be an arbitrary lifting of $f_{i}: S_{i} \rightarrow T_{i}$ to the universal covering such that $F_{i}(0)=0$. Let $\widetilde{G}_{i} \subset \Delta^{2}$ be the graph of $F_{i}$, which is a lifting of $G_{i} \subset S_{i} \times T_{i}$ to the universal cover $\Delta^{2}$ of $S_{i} \times T_{i}$. Since $F_{i}^{*} d s_{\Delta}^{2} \leq \mu_{i} \cdot d s_{\Delta}^{2}$ and $\mu_{i} \rightarrow 0$ as $i \rightarrow \infty$, the holomorphic maps $F_{i}: \Delta \rightarrow \Delta$ converge to a constant map $F_{\infty}, F_{\infty}(z)=0$ for any $z \in \Delta$. For $i \geq 1$, let $p_{i} \in \widetilde{G}_{i}$ be an arbitrary point. Equip $\Delta^{2}$ with the product metric $d s_{\Delta^{2}}^{2}$ given by the Poincaré metric $d s_{\Delta}^{2}$ in each factor, and denote by $B(p ; r)$ the geodesic ball centred at $p$ and of radius $r>0$ on $\left(\Delta^{2} ; d s_{\Delta}^{2}\right)$. Consider the sequence of complex submanifolds $\widetilde{G}_{i} \cap B\left(p_{i} ; 1\right) \subset B\left(p_{i} ; 1\right)$. Let $\varphi_{i} \in \operatorname{Aut}_{o}\left(\Delta^{2}\right)$ for which $\varphi_{i}\left(p_{i}\right)=0$. Then, $\varphi_{i}\left(\widetilde{G}_{i}\right) \subset \Delta^{2}$ is again the graph of a holomorphic mapping $F_{i}^{\prime}: \Delta \rightarrow \Delta$ which is a lifting of $f_{i}: S_{i} \rightarrow T_{i}$ satisfying $F_{i}^{\prime}(0)=0$. The convergence argument applies therefore equally to the sequence $F_{i}^{\prime}$ to show that $\varphi_{i}\left(\widetilde{G}_{i}\right) \subset B(0 ; 1)$ converge as complex-analytic subvarieties to $B_{1} \times\{0\}$, where $B_{1} \subset \Delta$ is the geodesic ball centred at 0 of radius 1 . It follows that $G_{i}$ is $\epsilon_{i}$-pinched with $\epsilon_{i}$ converging to 0 , and $G_{i} \subset S_{i} \times T_{i}$ is modelled on $\left(\Delta^{2} ; \Delta \times\{0\}\right)$ for $i$ sufficiently large, in the sense of Eyssidieux-Mok [EysMok1995]. In other words, gap rigidity fails for $\left(\Delta^{2} ; \Delta \times\{0\}\right)$.

Let us give the idea of the proof of Theorem 2. Let $f: S \rightarrow T$ be a generically $s$-to-1 holomorphic map between compact Riemann surfaces of genus $g(S), g(T) \geq 2$, and let $r$ be the degree of the ramification divisor. By the Riemann-Hurwitz formula we have $2(g(S)-1)=2 s(g(T)-1)+r$. Denote by $\|\cdot\|$ norms induced by the Poincaré metrics $d s_{S}^{2}$ resp. $d s_{T}^{2}$ on $S$ resp. $T$ of constant curvature -2 . Then, $\int_{S}\|d f\|^{2}=$ $s \cdot \operatorname{Area}\left(T, d s_{T}^{2}\right)$. It follows from the Gauss-Bonnet Theorem that the average of $\|d f\|^{2}$ over $S$ is $s \cdot \frac{\operatorname{Area}\left(T, d s_{T}^{2}\right)}{\operatorname{Area}\left(S, d s_{S}^{2}\right)}=s \cdot \frac{g(T)-1}{g(S)-1}$, which is $\frac{2 s(g(T)-1)}{2 s(g(T)-1)+r}$ by the Riemann-Hurwitz formula. If we fix $T$ and $s>1$ then this average would get arbitrarily small by choosing $r$ arbitrarily large. It is however not clear at all how sup $\|d f\|$ can be bounded in terms of the ramification divisor. We will fix some compact Riemann surface $T$ and take double covers, and our approach is to choose the branching locus to be more or less evenly spaced on $T$. One can most properly talk about an "evenly spaced" set of points on an elliptic curve by exploiting the group structure. For instance, for integers $n>1$ the set of $n$-torsion points can be considered such a set. We will take a ramified double cover $h: T \rightarrow E$ to get $T$ of genus $\geq 2$ and obtain $f_{i}: S_{i} \rightarrow T$ by taking double covers over $T$ such that the branching locus of $h \circ f_{i}: S_{i} \rightarrow E$ is evenly spaced in a precise way, allowing us to work on the elliptic curve $E$ by descent. In order to get an estimate on $\sup \left\|d f_{i}\right\|$ we will exploit the group structure on $E$ and the 
uniqueness properties of singular Hermitian metrics of negative Gaussian curvature on $E$ with prescribed (fractional) orders of poles at a finite number of points.

1.2. Proof of Theorem 2. We have the following standard Lemma.

Lemma 1. Let $C$ be a compact Riemann surface, $n$ be a positive integer, and $q_{1}, \cdots, q_{2 n}$ be distinct points on $C$. Then, there exists a compact Riemann surface $K$, and a branched double covering map $f: K \rightarrow C$ which is branched precisely over $q_{1}, \cdots, q_{2 n}$. In other words, $f^{-1}\left(q_{k}\right)=\left\{p_{k}\right\}$ and $f$ is precisely ramified (with ramification order 1 a fortiori) at the $2 n$ distinct points $p_{1}, \cdots, p_{2 n}$.

Proof. Let $[D]$ be the divisor line bundle of degree $2 n$ defined by the set $\left\{q_{1}, \ldots, q_{2 n}\right\}$ of simple points, and let $L$ be a holomorphic line bundle such that $L^{2} \cong[D] . \quad L$ exists because any point in $\operatorname{Pic}^{0}(C)$ is 2-divisible. Let now $s$ be the canonical section of the divisor line bundle $[D]$ and $t$ be the 2 -valent section of $\pi: L \rightarrow C$ over $C$ whose graph is defined by $K=\left\{v \in L: v^{2}=s(\pi(v))\right\}$. Then $K \subset L$ is nonsingular, and, putting $f=\left.\pi\right|_{K}$, we have a double cover $f: K \rightarrow C$ branched precisely over the $2 n$ distinct points $q_{1}, \cdots, q_{2 n}$.

We will now give a proof of Theorem 2 by constructing an example where all the target Riemann surfaces $T_{i}$ are identical.

Let $L \subset \mathbb{C}$ be any lattice and denote by $E=\mathbb{C} / L$ the quotient elliptic curve. Let $e \in E$ be a nonzero torsion point of order 2. By Lemma 1 there is a compact Riemann surface $T$ and a double cover $h: T \rightarrow E$ branched precisely over 0 and $e$. Write $q_{1}=$ $h^{-1}(0)$ and $q_{2}=h^{-1}(e)$. Let $m=2 i-1$ be an odd positive integer and consider the holomorphic map $\Phi_{m}: E \rightarrow E$ defined by $\Phi_{m}(x)=m x$. Let $D_{i}:=\Phi_{m}^{-1}(\{0, e\}) \subset E$. Note that $D_{1}=\{0, e\}$ and $\operatorname{Card}\left(D_{i}\right)=2 m^{2}$. Since $(m-1) e=i(2 e)=0$ on $E$, so that $m \cdot e=e$, we have also $0, e \in D_{i}$ for each positive integer $i$. Let $i \geq 2$, i.e., $m \geq 3$. Again by Lemma 1 there is a compact Riemann surface $S_{i}$ and a double cover $f_{i}: S_{i} \rightarrow T$ branched precisely over the reduced divisor $\Delta_{i}:=h^{-1}\left(D_{i}-D_{1}\right)$, noting that $\operatorname{Card}\left(\Delta_{i}\right)=4\left(m^{2}-1\right)$. We claim that $f_{i}: S_{i} \rightarrow T$ gives a sequence of holomorphic maps such that, writing $\mu_{i}=\sup \left\{\frac{f_{i}^{*} d s_{T}^{2}(x)}{d s_{S_{i}}^{2}(x)}: x \in S_{i}\right\}$, we have $\lim _{i \rightarrow \infty} \mu_{i}=0$.

We proceed to compare the Hermitian metrics $d s_{S_{i}}^{2}$ and $f_{i}^{*} d s_{T}^{2}$ by descending to the elliptic curve $E$. Since $h: T \rightarrow E$ is a double cover, the holomorphic mapping on $T-h^{-1}(\{0, e\})$ switching the two points of fibers of $h$ extends to an automorphism $\sigma$ of $T$ fixing the ramification points. $\sigma$ fixes the Poincaré metric $d s_{T}^{2}$ on $T$ of Gaussian curvature -2 . Hence, $d s_{T}^{2}$ descends to a Hermitian metric $\theta$ of Gaussian curvature -2 on $E-\{0, e\}$. Extending across the branching points 0 and $e, \theta$ can be interpreted as a Hermitian metric on $E$ with simple poles at 0 and $e$. More precisely, in terms of a local holomorphic coordinate $z$ at 0 or at $e$, we can write $\theta=\frac{a(z)}{|z|}|d z|^{2}$, where $a(z)$ is a continuous positive function which is smooth except possibly at $z=0$. We may say that $\theta$ is a Hermitian metric on the $\mathbb{Q}$-line bundle $T_{E} \otimes\left[D_{1}\right]^{-\frac{1}{2}}$. Similarly for $i>1$, by means of the double cover $f_{i}: S_{i} \rightarrow T$, the Poincaré metrics $d s_{S_{i}}^{2}$ of Gaussian curvature -2 descends to a Hermitian metric $\eta_{i}$ on the $\mathbb{Q}$-line bundle $T_{T} \otimes\left[\Delta_{i}\right]^{-\frac{1}{2}}$. We observe that $\eta_{i}$ is invariant under the holomorphic involution $\sigma$ on $T$. In fact $f_{i}^{*}\left(\sigma^{*} \eta_{i}\right)$ gives a smooth Hermitian metric on $S_{i}$ of constant Gaussian curvature -2 , so that $f_{i}^{*}\left(\sigma^{*} \eta_{i}\right)=d s_{S_{i}}^{2}$ by the uniqueness of Poincaré metrics of Gaussian curvature -2 , i.e., by the Ahlfors-Schwarz Lemma. It follows that $\sigma^{*} \eta_{i}=\eta_{i}$, as observed. As a consequence $\theta_{i}, i>1$, descends to a Hermitian metric $\theta_{i}$ on $E-D_{i}$. Across points 
of $D_{i}-D_{1}, \theta_{i}$ extends to a Hermitian metric with a simple pole since $h: T \rightarrow E$ is unramified over $\Delta_{i}=h^{-1}\left(D_{i}-D_{1}\right)$. On the other hand, $\theta_{i}$ extends across points of $D_{1}=\{0, e\}$ as a Hermitian metric with a simple pole because $\eta_{i}$ is smooth and positive at the two points $h^{-1}(0)=q_{1}$ and $h^{-1}(e)=q_{2}$, and $h$ is ramified at $q_{1}$ resp. $q_{2}$ to the order 1 . More precisely, $\theta_{i}$ is a Hermitian metric on the $\mathbb{Q}$-line bundle $T_{E} \times\left[D_{i}\right]^{-\frac{1}{2}}$ for $i>1$. We will also write $\theta_{1}$ for $\theta$, so that the last statement is true for $i \geq 1$.

For $i>1$ and for any $x \in S_{i}, \frac{f_{i}^{*} d s_{T}^{2}(x)}{d s_{S_{i}}^{2}(x)}$ is the same as $\frac{\theta_{1}(y)}{\theta_{i}(y)}$, where $y=h \circ f_{i}(x)$, provided that $y \notin D_{i}$. For such points $y \in E$ the inequality $f_{i}^{*} d s_{T}^{2}(x) \leq d s_{S_{i}}^{2}(x)$ translates into the inequality $\theta_{1}(y) \leq \theta_{i}(y)$. Since the endomorphism $\Phi_{m}: E \rightarrow E$ is unramified, $\Phi_{m}^{*}\left(\theta_{1}\right)$ gives a smooth Hermitian metric on the $\mathbb{Q}$-line bundle $T_{E} \otimes\left[D_{i}\right]^{-\frac{1}{2}}$. As in the last paragraph, by pulling-back to the double cover $R_{i}$ over $E$ branched precisely over $D_{m}$, Card $\left(D_{m}\right)=2 m^{2}$, from the uniqueness of the Poincaré metric of Gauss curvature -2 on $R_{i}$ it follows that $\theta_{i}=\Phi_{m}^{*} \theta_{1}$. We have therefore $\mu_{i}=$ $\sup \left\{\frac{\theta_{1}(y)}{\Phi_{m}^{*} \theta_{1}(y)}: y \notin D_{i}\right\}$, where $m=2 i-1$. It remains to show that $\mu_{i} \rightarrow 0$ as $i \rightarrow \infty$.

Fix a Euclidean metric $\omega$ on $E$ such that $\theta_{1}>\omega$. Then $\Phi_{m}^{*} \theta_{1}>m^{2} \cdot \omega$. Fix a coordinate unit disk $U_{1}$ at 0 (resp. $U_{2}$ at $e$ ), with coordinte $z$, so that $z(0)=0$ (resp. $z(e)=0$ ), and such that $\theta_{1}=\frac{a(z)}{|z|}|d z|^{2}$ with $a(z)$ a continuous positive function bounded between two positive constants. There is a positive constant $K$ such that $\theta_{1}(y) \leq K \omega$ for $y \in E-U_{1}-U_{2}$. Hence $\frac{\theta_{1}(y)}{\Phi_{m}^{*} \theta_{1}(y)}<\frac{K}{m^{2}}$ for $y \in E-U_{1}-U_{2}$. At $0 \in E$ we may choose $U_{1}$ such that $\Phi_{m}(z)=m \cdot z$ as a germ at 0 . For $|z|<\frac{1}{m}$, $\Phi_{m}^{*} \theta_{1}(z)=\frac{a(m z)}{|m \cdot z|} m^{2}|d z|^{2}=\frac{m \cdot a(m z)}{|z|}|d z|^{2}$, so that $\frac{\theta_{1}(z)}{\Phi_{m}^{*} \theta_{1}(z)}<\frac{a(z)}{m \cdot a(m z)}<\frac{C_{1}}{m}$ for some constant $C_{1}$. On the other hand, for $|z|>\frac{1}{m}$ we have $\theta_{1}(z)<C_{2} m \cdot \omega$ for some positive constant $C_{2}$, while $\Phi_{m}^{*} \theta_{1}(z)>m^{2} \cdot \omega$, so that $\frac{\theta_{1}(z)}{\Phi_{m}^{*} \theta_{1}(z)}<\frac{C_{2}}{m}$. We have therefore $\frac{\theta_{1}(z)}{\Phi_{m}^{*} \theta_{1}(z)}<\frac{C}{m}$ for $C=\max \left(C_{1}, C_{2}\right)$, for $z \in U_{1}$ and similarly for $z \in U_{2}$. Combining with the estimate on $E-U_{1}-U_{2}$, we have for $m=2 i-1$ sufficiently large the estimate $\frac{\theta_{1}(y)}{\Phi_{m}^{*} \theta_{1}(y)}<\frac{C}{m}$ for all $y \in E-D_{i}$. It follows that $\mu_{i}<\frac{C}{m} \rightarrow 0$ as $i \rightarrow \infty$. The proof of Theorems 1 and 2 is complete.

Remarks. We note that the unit disk plays a very special role in the proof of Theorem 1. The analogue of Theorem 1 does not apply when $\Delta$ is replaced by an irreducible bounded symmetric domain $D$ of rank $\geq 2$ (cf. (3.3), Proposition 4). The case where $D$ is of rank 1 but of dimension $\geq 2$ remains open.

\section{Gap rigidity in the Zariski topology by intersection theory.}

2.1. Basic facts and notations on geodesic embeddings of bounded symmetric domains.

2.1.1. Lie theoretic data attached to a symmetric domain. There is a 1-1 correspondence between bounded symmetric domain $(\Omega, o)$ and semisimple Lie algebras of Hermitian type. These are Lie theoretic data $\left(\mathfrak{g}, H_{0}\right),(\mathfrak{g}, \theta)$ a semisimple Lie algebra with a Cartan involution and $H_{0}$ an element of the center of the associated maximal compact subalgebra such that $\operatorname{ad}\left(H_{0}\right)^{2}=\theta$ (see [Sat1980]).

If $\Omega$ is irreducible, the correspondence can be described as follows: $\Omega=G / K$ and $o=e K$ where $G$ is the Lie group underlying the real points of a connected almost simple real algebraic group, also denoted by $G$, such that $G^{\mathbb{C}}=G \otimes_{\mathbb{R}} \mathbb{C}$ is connected and simply connected, $K$ is a maximal compact subgroup such that the 
center of $K$ is isomorphic to $U(1)$. The adjoint group of $G$ is the identity component of $\operatorname{Aut}(\Omega)$. Let $\mathfrak{g}$ be the Lie algebra of $G, \mathfrak{l}$ be the Lie algebra of $K, \theta$ the Cartan involution of the symetric pair $(G, K), \mathfrak{g}=\mathfrak{l} \oplus \mathfrak{p}$ the Cartan decomposition. We choose $H_{0} \in \mathfrak{z}$ satisfying $\operatorname{ad}\left(H_{0}\right)^{2}=\theta$, such that, when restricted to $\mathfrak{p}$, ad $\left(H_{0}\right)$ corresponds to the almost complex structure operator of $\Omega$ under the canonical isomorphism can : $T_{o}^{\mathbb{R}} \Omega \rightarrow \mathfrak{p}$. In particular $\mathfrak{p}^{\mathbb{C}}=\mathfrak{p}^{+} \oplus \mathfrak{p}^{-}$, where $\mathfrak{p}^{ \pm}$is the $\pm \sqrt{-1}$-eigenspace of $\operatorname{ad}\left(H_{0}\right)$ and $\mathfrak{p}^{+}$corresponds to $T^{1,0} \Omega$. Let $($. , . ) $\mathcal{K}$ be the Killing form on $\mathfrak{g}$. A $G$-invariant Riemannian metric $g_{o}^{\Omega}$ can be constructed such that $\left.g_{o}^{\Omega}\right|_{T_{o} \Omega}=\left.\operatorname{can}^{*}(., .)_{\mathcal{K}}\right|_{\mathfrak{p}}, g_{o}^{\Omega}$ is Kähler-Einstein.

A general bounded symetric domain $(\Omega, o)$ splits as a product of irreducible ones $\Omega=\Omega_{1} \times \ldots \times \Omega_{a}$ and we define $\mathfrak{g}=\mathfrak{g}_{1} \times \ldots \times \mathfrak{g}_{a}, H_{0}=\left(H_{0}\right)_{1} \times \ldots \times\left(H_{0}\right)_{a}$, $G_{0}=\left(G_{0}\right)_{1} \times \ldots \times\left(G_{0}\right)_{a}, K=K_{1} \times \ldots \times K_{a}$.

The converse correspondence constructing from the data $\left(\mathfrak{g}, H_{0}\right)$ as above a bounded symmetric domain in a complex vector space is a celebrated theorem of Harish-Chandra's, the classical cases being in E. Cartan's thesis.

The action of $K \subset G$ induced by the adjoint action of $G$ on $\mathfrak{g}$ leaves invariant the decomposition $\mathfrak{g}^{\mathbb{C}}=\mathfrak{p}^{+} \oplus \mathfrak{p}^{-} \oplus \mathbb{l}^{\mathbb{C}}$ and gives rise to the isotropy representation of $K$ on $T_{o}^{1,0} \Omega=\mathfrak{p}^{+}$.

2.1.2. Isotropy action on the Grassmannian of $\mathbf{p}$-planes in $\mathfrak{p}^{+}$. Consider $\operatorname{Gr}\left(p, T_{o} \Omega\right)$ the Grassmannian parametrizing complex $p$-planes in $\mathfrak{p}^{+}=T_{o}^{1,0} \Omega$.

We endow $\Lambda^{p} \mathfrak{p}^{+}$with the Hermitian metric functorially attached to the Hermitian metric on $\mathfrak{p}^{+}$defined by the formula $(\alpha, \beta)_{\mathfrak{p}^{+}}=(\alpha, \bar{\beta})_{\mathcal{K}}$. This defines a Fubini-Study metric on $\mathbb{P}\left(\Lambda^{p} \mathfrak{p}^{+}\right)$, which is Kähler. Consider the Kähler metric $d s_{p}^{2}$ on $\operatorname{Gr}\left(p, T_{o} \Omega\right)$ induced by this Fubini-Study metric under the Plücker embedding $\operatorname{Gr}\left(p, T_{o} \Omega\right) \rightarrow$ $\mathbb{P}\left(\Lambda^{p} \mathfrak{p}^{+}\right)$. Call this the Fubini-Study metric on $\operatorname{Gr}\left(p, T_{o} \Omega\right)$. The $K$-invariant Kähler metrics on $\operatorname{Gr}\left(p, T_{o} \Omega\right)$ take the form const.ds $s_{p}^{2}$, if $\Omega$ is irreducible.

Let $\mathcal{A} \in \operatorname{Gr}\left(p, T_{o} \Omega\right)$ be a $p$-plane in $\mathfrak{p}^{+}$. Let $B=\left(e_{1}, \ldots, e_{p}\right)$ be a unitary basis of $\mathcal{A}$. Let $S(B, \mathcal{A})=\sqrt{-1} \sum_{i=1}^{p}\left[e_{i}, \overline{e_{i}}\right]$, where $[-,-]$ is the Lie bracket in $\mathfrak{g}^{\mathbb{C}}$. This expression is obviously independant of $B$ and defines a real-analytic mapping $\Sigma: \operatorname{Gr}\left(p, T_{o} \Omega\right) \rightarrow \mathfrak{l}$.

2.1.3. Embeddings of symmetric domains. Consider two Lie algebras of Hermitian type $\left(\mathfrak{g}, H_{0}\right)$ and $\left(\mathfrak{g}^{\prime}, H_{0}^{\prime}\right)$. A $\left(H_{1}\right)$-homomorphism $\rho:\left(\mathfrak{g}, H_{0}\right) \rightarrow\left(\mathfrak{g}^{\prime}, H_{0}^{\prime}\right)$ is a Lie algebra morphism $\rho: \mathfrak{g}^{\prime} \rightarrow \mathfrak{g}$ such that $\operatorname{ad}\left(H_{0}\right) \rho=\rho \operatorname{ad}\left(H_{0}^{\prime}\right)$. An $\left(H_{2}\right)$-homomorphism is a Lie algebra morphism satisfying $\rho\left(H_{0}^{\prime}\right)=H_{0} . \quad\left(H_{2}\right)$ homomorphisms are $\left(H_{1}\right)$, see [Sat1980], pp. 83-88. These notions have been introduced by Satake [Sat1965].

Totally geodesic embeddings of pointed symmetric domains are in 1-1 correspondence with injective $\left(H_{1}\right)$-homomorphisms. Indeed, such a $\rho$ yields a morphism of the underlying symmetric Lie algebras that is complex linear for the complex structures on $\mathfrak{p}^{\prime}$ (resp. $\mathfrak{p}$ ) induced by $a d\left(H_{0}\right)$ and can be exponentiated to a homomorphism $\rho: G^{\prime} \rightarrow G$ satisfying $\rho\left(K^{\prime}\right)=K$, so that the map $G^{\prime} / K^{\prime} \rightarrow G / K$ is holomorphic. Those corresponding to $\left(H_{2}\right)$-homomorphisms will be called $\left(H_{2}\right)$.

Lemma 2. Let $\Omega$ be irreducible. Then there exists a real number $c_{\Omega}$ such that $\Sigma\left(\mathfrak{p}^{+}\right)=\sqrt{-1} c_{\Omega} H_{0}$.

Proof. The lemma follows directly from the independence of $\Sigma$ with the respect to the choice of a basis. Indeed, we may use a change of basis which lies in $K$ to see that $\Sigma$ is a fixed point of the adjoint action of $K$. Hence the lemma. 
Let $\Omega^{\prime}$ be an irreducible subdomain of $\Omega$ and let $\rho: \mathfrak{g}^{\prime} \rightarrow \mathfrak{g}$ be the corresponding $\left(H_{1}\right)$-embedding. Let $d_{\Omega^{\prime}, \Omega}>0$ be the real number defined by $\left.g_{o}^{\Omega}\right|_{\Omega^{\prime}}=d_{\Omega^{\prime}, \Omega} \cdot g_{o}^{\Omega^{\prime}}$.

In order to state our results on gap rigidity in the Zariski topology, we need to introduce a variant of the $\left(H_{1}\right)$ and $\left(H_{2}\right)$ conditions. Consider an embedding of bounded symmetric domains $j: \Omega^{\prime} \rightarrow \Omega$ and let $\rho: \mathfrak{g}^{\prime} \rightarrow \mathfrak{g}$ be the corresponding $\left(H_{1}\right)$-embedding. Let $\Omega^{\prime}=\Omega_{1}^{\prime} \times \ldots \times \Omega_{a}^{\prime}$ be the irreducible decomposition of $\Omega^{\prime}$. We will say $j$, resp. $\rho$, is $\left(H_{3}\right)$ iff the following holds:

$$
\rho\left(\sum_{i=1}^{a} c_{\Omega_{i}^{\prime}} d_{\Omega_{i}^{\prime}, \Omega} H_{0 i}^{\prime}\right) \in \mathbb{R} H_{0} .
$$

LEMMA 3. ( $\left.H_{3}\right)$-embeddings are $\left(H_{2}\right)$. An $\left(H_{2}\right)$-embedding $\Omega^{\prime} \rightarrow \Omega$ is $\left(H_{3}\right)$ iff the Einstein constants of the metrics $\left.g_{o}^{\Omega}\right|_{\Omega_{i}^{\prime}}$ are the same, where $\Omega^{\prime}=\Omega_{1}^{\prime} \times \ldots \times \Omega_{a}^{\prime}$ and $\Omega_{i}^{\prime}$ is irreducible.

Proof. Let $H_{1}^{\prime}=A . \sum_{i=1}^{a} c_{\Omega_{i}^{\prime}} d_{\Omega_{i}^{\prime}, \Omega} H_{0 i}^{\prime}, A$ a constant such that $\rho\left(H_{1}^{\prime}\right)=H_{0}$. For every $z \in \mathfrak{g}^{\prime}$, we have $\left[H_{0}-\rho\left(H_{0}^{\prime}\right)^{2}, \rho(z)\right]=0$ since $\rho$ is $\left(H_{1}\right)$, hence $\left[\rho\left(H_{1}^{\prime}-H_{0}^{\prime}\right), \rho(z)\right]=$ 0 and $\left[H_{1}^{\prime}-H_{0}^{\prime}, z\right]=0$. Since $\mathfrak{g}^{\prime}$ is semisimple this implies that $H_{1}^{\prime}=H_{0}^{\prime}$. A fortiori, $\rho$ is $\left(H_{2}\right)$. It also follows that for every $1 \leq i \leq a, A \cdot c_{\Omega_{i}^{\prime}} d_{\Omega_{i}^{\prime}, \Omega}=1$. A standard curvature formula implies that $c_{\Omega_{i}^{\prime}} d_{\Omega_{i}^{\prime}, \Omega}$ is minus the Einstein constant of $\left.g_{o}^{\Omega}\right|_{\Omega_{i}^{\prime}}$.

In particular, for an embedding of irreducible bounded symmetric domains $\Omega^{\prime} \subset$ $\Omega$, the $\left(H_{2}\right)$ and $\left(H_{3}\right)$ conditions are equivalent.

2.2. Statement of main results. The purpose of this section is to give a general criterion under which gap rigidity holds for a pair $(\Omega, D)$ in the Zariski topology with $\Omega$ irreducible (and of rank $\geq 2$ ).

Let us first state a GIT interpretation of the $\left(H_{3}\right)$-condition. We say that $\mathcal{A} \in$ $\operatorname{Gr}\left(p, T_{o} \Omega\right)$ is GIT-semistable if there is a $K$-invariant complex closed hypersurface $\mathcal{Z}_{o} \subset \operatorname{Gr}\left(p, T_{o} \Omega\right)$ such that $\mathcal{A} \notin \mathcal{Z}_{o}$.

Proposition 1. The embeddings of symmetric domains $\left(\Omega^{\prime}, o\right) \rightarrow(\Omega, o)$ such that $T_{o} \Omega^{\prime}$ is GIT-semistable in $\operatorname{Gr}\left(\operatorname{dim} \Omega^{\prime}, T_{o} \Omega\right)$ with respect to the isotropy action of $K$ are precisely the $\left(H_{3}\right)$-embeddings.

As a step towards his classification of embeddings of symmetric domains, Ihara [Iha1967] obtained the full classification theory of $\left(\mathrm{H}_{2}\right)$-embeddings.

It turns out that, thanks to Proposition 1, images of $\left(H_{3}\right)$-embeddings of bounded symmetric domains play an important role in the question of gap rigidity in the Zariski topology. A compact totally-geodesic complex submanifold $S$ of a quotient $X$ of a bounded symmetric domain by a discrete group of biholomorphic automorphisms will be referred to as a holomorphic geodesic cycle. If $S \subset X$ arises from an $\left(H_{3}\right)$ embedding, $S$ will be referred to as an $\left(H_{3}\right)$-holomorphic geodesic cycle. We prove:

Theorem 3. Let $\Omega=G / K$ be an $n$-dimensional irreducible bounded symmetric domain. Let $\Gamma$ be a torsion-free discrete group of biholomorphic automorphisms of $\Omega$, and write $X:=\Omega / \Gamma$. Assume there exists $D \subset \Omega$ an $\left(H_{3}\right)$-embedding, $o \in D$, $\operatorname{dim}(D):=p$.

Fix a projective $K$-invariant hypersurface $\mathcal{Z}_{o} \subset \operatorname{Gr}\left(p, T_{o} \Omega\right)$ such that $\left[T_{o}(D)\right] \notin$ $\mathcal{Z}_{o}$. Denote by $\pi: \mathbb{G}(X) \rightarrow X$ the Grassmann bundle of p-dimensional tangent planes and write $\mathcal{Z}=\mathcal{Z}_{X} \subset \mathbb{G}(X)$ for the locally homogeneous subbundle of projective hypersurfaces corresponding to $\mathcal{Z}_{o}$. 
Let $S \subset X$ be a compact complex p-dimensional submanifold such that for any $x \in S,\left[T_{x}(S)\right] \notin \mathcal{Z}_{x}$. Then, $S \subset X$ is an $\left(H_{3}\right)$-holomorphic geodesic cycle.

Remark. We do not exclude the possibility that $S$ is uniformized by some $\left(H_{3}\right)$ complex totally-geodesic submanifold $D^{\prime} \subset \Omega$ which is not equivalent to $D$ under $\operatorname{Aut}(\Omega)$. This may in fact happen, but there are up to equivalence under Aut $(\Omega)$ only a finite number of possibilities.

\subsection{Proof of Proposition 1.}

2.3.1. Moment map of the isotropy action on the Grassmannian of planes in $\mathfrak{p}^{+}$. Let $\omega_{p}$ be the Kähler form associated to $d s_{p}^{2}$. View it as a symplectic form. $K$ acts on the symplectic manifold $\left(\operatorname{Gr}\left(p, T_{o} \Omega\right), \omega_{p}\right)$ preserving the symplectic form. Since the Grassmannian is simply connected, there exists a moment map $\mu_{p}$ for this symplectic action [Sou1970] (see [MumFogKir1994], Chap. 8).

It might be useful to recall the definition of this central concept of symplectic geometry. Let $(S, \omega)$ be symplectic manifold acted upon by a connected Lie group $M$ whose Lie algebra is denoted by $\mathfrak{m}$, a map $\mu: S \rightarrow \mathfrak{m}^{*}$ is called a moment map if it is smooth, $M$-equivariant with respect to the given action of $M$ on $X$ and the coadjoint action and

$$
\forall x \in M, \xi \in T_{x} S, l \in \mathfrak{m}, \quad<<d \mu(x), \xi>, l>=<\omega(x), \xi \wedge l_{x}>
$$

where $\langle-,->$ denotes the canonical pairing between a vector space $E$ and its dual $E^{*}$ and $l_{x}=\left.\frac{d}{d t} \exp (t l) x\right|_{t=0}$.

When it exists, a moment map is unique up to the addition of a coadjoint fixed point in $\mathfrak{m}^{*}$. When $M=\mathbb{R}$, a moment map is a global hamiltonian function for the flow of $\frac{d}{d t}$.

If $V$ is a complex vector space with a non degenerate sesquilinear pairing $h$, we also denote by $h$ the corresponding conjugate linear isomorphism $V \rightarrow V^{*}$. In the next lemma, $V=\mathfrak{l}^{\mathbb{C}}$ and $h_{\mathfrak{g}, \mathfrak{l}}(\lambda, \mu)=(\lambda, \bar{\mu})_{\mathcal{K}}$.

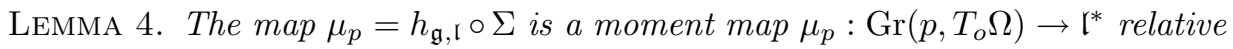
to the symplectic action of $K$ on $\left(\operatorname{Gr}\left(p, T_{o} \Omega\right), \omega_{p}\right)$.

\subsubsection{Proof of Lemma 4 .}

LEMMA 5. Let the Lie group $L_{1} \times L_{2}$ act symplectically on $(S, \omega)$ with moment map $\mu=\left(\mu_{1}, \mu_{2}\right): S \rightarrow \mathfrak{l}_{1}^{*} \times \mathfrak{l}_{2}^{*}$. Let $z$ be a coadjoint fixed point of $L_{2}$ which is furthermore a regular value of $\mu_{2}$ and assume $L_{1}$ acts freely on $\mu_{2}^{-1}(z)$. The symplectic quotient [MarWei1974] $S / / L_{2}=\mu_{2}^{-1}(z) / L_{2}$ is acted upon symplectically by $L_{1}$. Let $\pi: \mu_{2}^{-1}(z) \rightarrow S / / L_{2}$ be the canonical quotient map.

The symplectic action of $L_{1}$ on $S / / L_{2}$ admits a moment map $\mu$ defined by the relation $\mu \circ \pi=\left.\mu_{1}\right|_{\mu_{2}^{-1}(z)}$.

Proof. $L_{1}$-equivariance is clear. Recall (see [MumFogKir1994], p. 146) that $\mu_{2}^{-1}(z)$ is a coisotropic submanifold of $S$ whose isotropic foliation $\operatorname{ker}\left(\left.\omega\right|_{\mu_{2}^{-1}(z)}\right)$ is precisely the foliation by $L_{2}$-orbits and that the symplectic form on $S / / L_{2}$ is the form induced by $\omega$ on local transversal sections of $\pi$. The required differential identity follows then directly from the definitions. 
An easy example is the moment map $\mu_{n}$ associated with the action of $U(n)$ on $\mathbb{C}^{n}$ with coordinates $\left(z^{i}\right)$ equipped with $\frac{\sqrt{-1}}{2 \pi} \sum_{i} d z^{i} \wedge d \bar{z}^{i}$. Identifying $\mathfrak{u}(n)$ and its dual by means of the scalar product $(x, y)_{\mathfrak{u}(n)}=-\operatorname{tr}(x y)$. We have:

$$
\mu_{n}\left(\left(z^{1}, \ldots, z^{n}\right)\right)=-\frac{\sqrt{-1}}{2 \pi}\left(z^{i} \bar{z}^{j}\right)_{1 \leq i, j \leq n} .
$$

Since the moment map for the action of $K$ on a product $X \times Y$ of two symplectic $K$-manifolds is the sum of the moment maps for $X$ and $Y$, it follows that the moment map for the action of $U(n) \times U(p)$ on complex matrices $Z=\left(z_{l}^{i}\right)_{1 \leq i \leq n, 1 \leq l \leq p}$ with $n$ rows and $p$ columns equipped with the Hermitian scalar product $\bar{Z} \mapsto \frac{1}{\pi} \operatorname{Tr}\left(Z^{t} \bar{Z}\right)$ is $\mu(Z)=-\frac{\sqrt{-1}}{2 \pi}\left(Z^{t} \bar{Z},-{ }^{t} \bar{Z} Z\right)=-\frac{\sqrt{-1}}{2 \pi}\left(\left(\sum_{l=1}^{p} z_{l}^{i} \bar{z}_{p}^{j}\right),-\left(\sum_{i=1}^{n} z_{l}^{i} \bar{z}_{m}^{i}\right)\right)$.

If $p \leq n$ the Grassmannian of $p$-planes in $\mathbb{C}^{n}$ equipped with its Fubini-Study 2-form is the symplectic quotient $M_{n, p}$ by $U(p)$ corresponding to the regular value $\frac{\sqrt{-1}}{2 \pi} I d_{p}$.

This gives the expression $\mu([Z])=-\frac{\sqrt{-1}}{2 \pi} Z^{t} \bar{Z}$ for any matrix $Z$ representing a unitary basis of $[Z]$ in this Grassmannian.

Let us restate this fact in slightly more invariant terms. Let $(V, h)$ be a Hermitian vector space. Use the canonical complex linear identifications $\mathfrak{u}(V, h)^{\mathbb{C}}=\operatorname{End}(V) \simeq$ $V \otimes V^{*}, \mathfrak{u}^{*}(V, h)^{\mathbb{C}} \simeq V^{*} \otimes V$ and the conjugate linear isomorphism $h: V \rightarrow V^{*}$. Let $\mathcal{A}$ be a $p$-plane in $V$ with unitary basis $\left(v_{1}, \ldots, v_{p}\right)$. The value at $\mathcal{A}$ of the moment map with respect to the Fubini-Study metric is $\mu_{p}(\mathcal{A})=\frac{\sqrt{-1}}{2 \pi} \sum_{i=1}^{p} h\left(v_{i}\right) \otimes v_{i}$.

Obviously, if $(S, \omega)$ is symplectic manifold acted upon by a connected Lie group $M$ admitting a moment map and $M^{\prime} \subset M$ is a Lie subgroup the moment map for $M^{\prime}$ is the composition of the moment map for $M$ with the canonical map $\mathfrak{m}^{*} \rightarrow\left(\mathfrak{m}^{\prime}\right)^{*}$.

We now compute the map $\mathfrak{p}^{+*} \otimes \mathfrak{p}^{+} \simeq \mathfrak{u}^{*}\left((-,-)_{\mathfrak{p}^{+}}\right)^{\mathbb{C}} \rightarrow \mathfrak{l}^{\mathbb{C} *}$ induced by the isotropy representation $\rho: K \rightarrow U\left((-,-)_{\mathfrak{p}^{+}}\right)$. Fix $v \in \mathfrak{p}^{+}, l \in \mathfrak{l}$ and $\left(e_{i}\right)$ a unitary basis of this vector space. We have:

$$
\begin{aligned}
<h_{\mathfrak{p}^{+}}(v) \otimes v, \rho(l)> & =<h_{\mathfrak{p}^{+}}(v) \otimes v, \sum_{i}\left[l, e_{i}\right] \otimes h_{\mathfrak{p}^{+}}\left(e_{i}\right)> \\
& =\sum_{i}<h_{\mathfrak{p}^{+}}(v),\left[l, e_{i}\right]><v, h_{\mathfrak{p}^{+}}\left(e_{i}\right)> \\
& =<h_{\mathfrak{p}^{+}}(v),[l, v]>=([l, v], v)_{\mathfrak{p}^{+}}=([l, v], \bar{v})_{\mathcal{K}} \\
& =(l,[v, \bar{v}])_{\mathcal{K}} .
\end{aligned}
$$

The last equality holds since the adjoint representation acts by isometries of the Killing form. This concludes the proof of Lemma 4.

\subsubsection{GIT analysis of embeddings of bounded symmetric domains.}

Semistability of $\left(H_{3}\right)$-embeddings. Lemma 4, Lemma 2 above and Kempf-Ness theory, a classical relation between moment maps and Geometric Invariant Theory (Theorem 8.3 of [MumFogKir1994]), imply

Corollary 1. Let $\mathcal{A} \in \operatorname{Gr}\left(p, T_{o} \Omega\right)$ such that $\Sigma(\mathcal{A})=c H_{0}$. Then $\mathcal{A}$ is GIT semistable with respect to the action of $K^{\mathbb{C}}$.

The $K$-action reduces to a $K / U(1)$ on the Grassmannian because $U(1)$ acts by complex homotheties on $\mathfrak{p}^{+}$. In particular the moment map composed with the projection to $\mathfrak{u}(1)^{*}$ is constant. So that the condition $\Sigma(\mathcal{A})=c H_{0}$ means the vanishing of the moment map for $K / U(1)$ on $\operatorname{Gr}\left(p, T_{o} \Omega\right)$. 
Alternatively, the values of the moment map are in a fixed affine hyperplane $H$ of the form $H_{c}=\left\{\zeta \in \mathfrak{I}^{*}\right.$, such that $\left.\left\langle\zeta, H_{0}\right\rangle=c\right\}$, where $c$ is a constant. Using Lemma 4 , we see that for an aribitrary subspace $\mathcal{B}, \Sigma(\mathcal{B})=c H_{0}+\Sigma^{\prime}(\mathcal{B})$ with $h_{\mathfrak{g}, \mathfrak{l}}\left(H_{0}, \Sigma^{\prime}(\mathcal{B})\right)=0$. For future use, we record the following observation

Lemma 6. Let $\mathcal{A} \in \operatorname{Gr}\left(p, T_{o} \Omega\right)$ such that $\Sigma(\mathcal{A})=c H_{0} .\|\Sigma\|^{2}=\|\Sigma\|_{\mathfrak{g}, \mathrm{l}}^{2}$ achieves its absolute minimum at $\mathcal{A}$.

Corollary 1 gives one implication in Proposition 1. We now prove the converse statement.

Embeddings of symmetric domains as critical points of the 'Morse function' attached to the moment map. Lemma 7 . Let $\left(\Omega^{\prime}, o\right) \rightarrow(\Omega, o)$ be a geodesic embedding. Then $\left[\rho\left(\mathfrak{p}^{+}\right)\right]$is a critical point of $\|\Sigma\|^{2}$.

Proof. The tangent space at $\mathcal{A} \in \operatorname{Gr}\left(p, T_{o} \Omega\right)$ is isometric to $H=H o m\left(\mathcal{A}, \mathcal{A}^{\perp}\right)$. If $v \in H$ and $\left(e_{i}\right)$ is a unitary basis of $\mathcal{A},\left(e_{i}+t . v\left(e_{i}\right)\right)$ is unitary up to the second order term in $t$, so that $\partial_{v}\|\Sigma(\mathcal{A})\|^{2}=\sum_{i j}\left(\left[v\left(e_{i}\right), \bar{e}_{i}\right],\left[e_{j}, \bar{e}_{j}\right]\right)+(i \leftrightarrow j)=2 \sum_{i}\left(v\left(e_{i}\right),\left[\Sigma, e_{i}\right]\right)$. Hence $\mathcal{A}$ is critical iff $[\Sigma(\mathcal{A}), \mathcal{A}] \in \mathcal{A}$. When $\mathcal{A}=\rho\left(\mathfrak{p}^{\prime+}\right)$ we have actually $\forall \alpha, \beta, \gamma \in$ $\mathcal{A},[[\alpha, \bar{\beta}], \gamma] \in \mathcal{A}$.

Conclusion of the proof. Kempf-Ness theory can be made more precise than Theorem 8.3 of [MumFogKir1994]. The statement we need is thm 8.10 p. 109 in [Kir1984] through the following consequence:

Proposition 2. . Let $K$ be a compact Lie group. Assume $K^{\mathbb{C}}$ acts linearily on a projective manifold $X$ embedded in $\mathbb{P}^{N}$. Let $\mu_{\mathbb{P} U(N+1)}: X \rightarrow \mathfrak{s u}(N+1)^{*}$ be the moment map corresponding to the Fubini-Study metric and $\mu: X \rightarrow \mathfrak{l}^{*}$ be the moment map obtained by composing $\mu_{\mathbb{P} U(N+1)}$ and the canonical surjection $\mathfrak{s u}(N+1)^{*} \rightarrow \mathfrak{l}^{*}$.

The set of semistable points of $X$ is the minimal Morse stratum, that is the set of points attracted by $\mu^{-1}(0)$ under the steepest descent flow of $\|\mu\|^{2}$.

In particular, every critical point of $\|\mu\|^{2}$ whose critical value is not 0 is unstable in the GIT sense.

Since the moment map we use has the required form, applying Lemma 7, we deduce Proposition 1.

2.4. Proof of Theorem 3. Denote also by $\pi: \mathbb{G}(\Omega) \rightarrow \Omega$ the Grassmann bundle of $p$-planes in $T(\Omega)$, and by $\mathcal{Z}_{\Omega} \subset \mathbb{G}(\Omega)$ the homogeneous bundle of projective hypersurfaces corresponding to $\mathcal{Z}_{o} \subset \operatorname{Gr}\left(p, T_{o}(\Omega)\right)=\mathbb{G}_{o}(\Omega)$. Let $\Omega \subset M$ be the Borel embedding of $\Omega$ into its compact dual $M=G_{c} / K$, and $N \supset D$ be the compact dual of $D$. $N$ is a complex submanifold of $M$. The complexification $K^{\mathbb{C}}$ of $K$ acts naturally as a group of automorphisms on $M$. Since $\mathcal{Z}_{o} \subset \mathbb{G}_{o}(\Omega)$ is complex-analytic, it is invariant under $K^{\mathbb{C}}$ and the image of $\mathcal{Z}_{o} \subset \mathbb{G}_{o}(\Omega)$ under the action of $\operatorname{Aut}(M)=G^{\mathbb{C}}$ defines a holomorphic subbundle $\mathcal{Z}_{M} \subset \mathbb{G}(M)$ of the Grassmann bundle of $p$-planes in $M$. $\mathcal{Z}_{\Omega}$ is the restriction of $\mathcal{Z}_{M}$ to $\Omega$, and it descends to $\mathcal{Z}=\mathcal{Z}_{X} \subset \mathbb{G}(X)$ under the action of $\Gamma$. The hypersurface $\mathcal{Z}_{M} \subset \mathbb{G}(M)$ defines a $K$-invariant divisor line bundle. $\mathbb{G}(M)$ embeds canonically into $\mathbb{P}\left(\Lambda^{p} T_{M}\right)$, where at each base point $x \in M$ the embedding $\mathbb{G}_{o}(M) \subset \mathbb{P}\left(\Lambda^{p} T_{o}(M)\right)$ is congruent to the Plücker embedding. The tautological line bundle $L$ on $\mathbb{P}\left(\Lambda^{p} T_{M}\right)$ restricts to the tautological line bundle on $\mathbb{G}(M)$, to be denoted also by $L$. Since the Picard group of both $M$ and the typical fiber $\mathbb{G}_{O}$ of $\pi: \mathbb{G}(M) \rightarrow M$ are both infinite cyclic, the Picard group of the total space $\mathbb{G}(M)$ is isomorphic to $\mathbb{Z}^{2}$, and is generated as a group by $\pi^{*} \mathcal{O}(1)$ and by $L$, where $\mathcal{O}(1)$ 
denotes the positive generator of $\operatorname{Pic}(M)$. Thus there exist positive integers $m, \ell$ such that $\mathcal{Z} \subset \mathbb{G}(M)$ is the zero-set of a $G^{\mathbb{C}}$-invariant ${ }^{1}$ section $s \in \Gamma\left(M, L_{M}^{-m} \otimes \pi^{*} \mathcal{O}(\ell)\right)$.

With this set-up the proof of [Mok2002], Theorem 1, where an argument in (1.2) using duality between Hermitian symmetric manifolds of compact and noncompact type was used, generalizes to complete the proof of Theorem 1 . The starting point is the Poincaré-Lelong equation on the Hermitian symmetric manifold of compact type $M$. Letting $g_{c}$ be a canonical Kähler-Einstein metric on $M, \widehat{g}_{c}$ the induced Hermitian metric on the tautological line bundle, $h_{c}$ be a $G_{c}$-invariant Hermitian metric on $\mathcal{O}(1)$, we have, by the Poincaré-Lelong equation

$$
\frac{\sqrt{-1}}{2 \pi} \partial \bar{\partial} \log \|s\|_{c}^{2}=m c_{1}\left(L, \widehat{g}_{c}\right)-\ell c_{1}\left(\pi^{*} \mathcal{O}(1), \pi^{*} h_{c}\right)+\left[\mathcal{Z}_{M}\right]
$$

(There was a sign mistake on the curvature term in the Poincaré-Lelong equation in [[Mok2002],(1.2) and the proof of Theorem 1] but they do not affect the rest of the argument.) Let now $\widehat{N} \subset \mathbb{G}(M)$ be the canonical lifting of the totally-geodesic submanifold $N \subset M$ to the Grassmann bundle $\mathbb{G}(M)$. Let $\omega_{c}$ be the Kähler form of $\left(M, g_{c}\right)$. Then, from $\widehat{N} \cap \mathcal{Z}_{M}=\emptyset$ and Stokes' Theorem we have

$$
\int_{\widehat{N}}\left(m c_{1}\left(L, \widehat{g}_{c}\right)-\ell c_{1}\left(\pi^{*} \mathcal{O}(1), \pi^{*} h_{c}\right)\right) \wedge\left(\pi^{*} \omega_{c}\right)^{p-1}=0,
$$

Denote by $\sigma: N \rightarrow \mathbb{G}(M)$ the canonical lifting map whose image is precisely $\widehat{N}$. We note that $\sigma^{*}\left(L, \hat{g}_{c}\right)$ is nothing other than $\left(K_{N}, \operatorname{det}\left(\left.g_{c}\right|_{N}\right)\right)$. In fact there is a correspondence between the vector bundle $\Lambda^{p} T_{N}$ over $N$ and the tautological line bundle $L$ over $\mathbb{P}\left(\Lambda^{p} T_{N}\right)$, which sends a vector $\eta \in \Lambda^{p} T_{x}(N)$ to the vector, also denoted by $\eta$, as an element over the point $[\eta]$. In this tautological identification the length of vectors is preserved, so that $\sigma^{*}\left(L, \hat{g}_{c}\right)$ is nothing other than $\left(K_{S_{c}}^{-1}, \operatorname{det}\left(\left.g\right|_{S_{c}}\right)\right)$, as asserted. $\omega_{c}$ is a positive multiple of $c_{1}\left(\mathcal{O}(1), h_{c}\right)$ and we may take the two to be the same in what follows. Then,

$$
\begin{aligned}
0 & =\int_{\widehat{N}}\left(m c_{1}\left(L, \widehat{g}_{c}\right)-\ell c_{1}\left(\mathcal{O}(1), \pi^{*} h_{c}\right)\right) \wedge\left(\pi^{*} \omega_{c}\right)^{p-1} \\
& =\int_{N}\left(m c_{1}\left(K_{N}^{-1}, \operatorname{det}\left(\left.g_{c}\right|_{N}\right)\right)-\ell c_{1}\left(\mathcal{O}(1), h_{c}\right)\right) \wedge \omega_{c}^{p-1} \\
& \left.=\int_{N} m \operatorname{Ric}\left(\left.g\right|_{N}\right)-\ell c_{1}\left(\mathcal{O}(1), h_{c}\right)\right) \wedge \omega_{c}^{p-1} \\
& =\int_{N}\left(\frac{m}{p} K\left(\left.g_{c}\right|_{N}\right)-\ell\right) \omega_{c}^{p}
\end{aligned}
$$

where $K\left(g_{c} \mid N\right)$ stands for the scalar curvature, which is a constant, which forces $K\left(\left.g_{c}\right|_{N}\right)=\frac{p \ell}{m}$. Denote by $g$ the Kähler-Einstein metric on $\Omega$ dual to $g_{c}$, etc., and $(E, h)$ the negative line bundle on $\Omega$ dual to $\left(\mathcal{O}(1), h_{c}\right)$. By our choice of $h_{c}$ we have $c_{1}(E, h)=-\omega$. For the same section $s \in \Gamma\left(\mathbb{G}(M), L \otimes \pi^{*} E\right)$, restricted to $\Omega$, denote by $\|s\|$ the norm measured in terms of $g$ and $h$. Then, we have the Poincaré Lelong equation on $\Omega$

$$
\frac{\sqrt{-1}}{2 \pi} \partial \bar{\partial} \log \|s\|^{2}=m c_{1}(L, \widehat{g})-\ell c_{1}\left(\pi^{*} E, \pi^{*} h\right)+\left[\mathcal{Z}_{\Omega}\right]
$$

\footnotetext{
${ }^{1}$ Note that $G^{\mathbb{C}}$ is unimodular.
} 
Since $s$ is $G^{\mathbb{C}}$ invariant, every ingredient of this Poincaré-Lelong equation descends to $\mathbb{G}(\Omega) / \Gamma$ and gives rise to a relation between cohomology classes on this manifold:

$$
\left[\mathcal{Z}_{\Omega / \Gamma}\right]=-m c_{1}(L)_{\Omega / \Gamma}+\ell c_{1}\left(\pi^{*} E_{\Omega / \Gamma}\right)
$$

Suppose now we have a compact totally-geodesic complex submanifold $S_{o} \subset X:=$ $\Omega / \Gamma$ uniformized by $D \subset \Omega$. Then, $\widehat{S}_{o} \cap \mathcal{Z}_{X}=\phi$ and we have

$$
\begin{aligned}
0 & =\int_{\widehat{S}_{o}}\left(m c_{1}(L, \widehat{g})-\ell c_{1}\left(E, \pi^{*} h\right)\right) \wedge\left(\pi^{*} \omega\right)^{p-1} \\
& =\int_{S_{o}}\left(m c_{1}\left(K_{S_{o}}^{-1}, \operatorname{det}\left(\left.g\right|_{S_{o}}\right)\right)-\ell c_{1}(E, h)\right) \wedge \omega^{p-1} \\
& \left.=\int_{S_{o}} m \operatorname{Ric}\left(\left.g\right|_{S_{o}}\right)-\ell c_{1}(E, h)\right) \wedge \omega^{p-1} \\
& =\int_{S_{o}}\left(\frac{m}{p} K\left(\left.g\right|_{S_{o}}\right)+\ell\right) \omega^{p}
\end{aligned}
$$

Since $K\left(\left.g\right|_{S_{o}}\right)=-K\left(\left.g_{c}\right|_{N}\right)$ the integrand vanishes identically on $S_{o}$, which has to be the case a priori. But we are going to characterize $p$-dimensional compact complex submanifolds $S \subset X$ whose tangent spaces do not belong to $\mathcal{Z}_{X}$. For the proof we do not assume the existence of $S_{o}$. Integrating the restriction of the Poincaré-Lelong equation to $S$ and assuming that $\widehat{S} \cap \mathcal{Z}_{X}=\phi$ we conclude that

$$
\int_{S}\left(\frac{m}{p} K\left(\left.g\right|_{S}\right)+\ell\right) \omega^{p}=0
$$

Once we have the intepretation of the integral over $\widehat{S}$ as an integral over $S$, Theorem 3 follows from [Eys1997], Proposition 9.2.5. The rest of the argument is actually a standard generalization of the proof of the Arakelov inequality, which we include for the sake of completeness. Indeed, the scalar curvature of $D$ is $K\left(\left.g\right|_{D}\right)=-C\left\|\Sigma\left(T_{o} D\right)\right\|^{2}$ by standard curvature formulas (see e.g. [Eys1997] p. 205-206), $C$ being a positive constant, and the scalar curvature of $S$ at $x$ is $K\left(\left.g\right|_{S}\right)_{x}=-C\left\|\Sigma\left(T_{x} S\right)\right\|^{2}-\left\|\sigma_{x}\right\|^{2}$ where $\sigma$ is the second fundamental form of the embedding $S \subset \Omega / \Gamma$. By Lemma 6 we have, at any point $x, \frac{m}{p} K\left(\left.g\right|_{S}\right)_{x}+\ell \leq-\frac{m}{p}\left\|\sigma_{x}\right\|^{2}$. Since the integral of the l.h.s. is 0 , we deduce that $\sigma_{x}=0$, i.e. $S$ is totally geodesic. This concludes the proof of Theorem 3.

Remarks. (a) It was convenient to make use of the compact dual and duality to check that for $s \in \Gamma\left(\mathbb{P}\left(T_{\Omega}\right), L^{-m} \otimes \pi^{*}(E)\right)$, the ratio of $m$ to $\ell$ is the right one. The same thing can be obtained by working on $\Omega$ alone, provided that we assume the fact that $D$ admits a torsion-free cocompact lattice, a result dating back to Borel ([Bor1963], Corollary to Theorem A).

(b) Modifying the above proof with a brute force curvature computation of the appropriate line bundle would also allow us to prove Theorem 3 for reducible domains $\Omega$. We did not feel compelled to give the full argument for such a slight generalization which we nevertheless have to mention.

2.5. Explicit examples. In this section, we give some examples of pairs $(\Omega, D)$ of bounded symmetric domains, $\Omega$ irreducible, $\Omega=G / K, \operatorname{dim}(D)=p$, such that 
$\left[T_{o}(D)\right]$ is a semistable point in the Grassmannian $\operatorname{Gr}\left(p, T_{o}(\Omega)\right)$ of $p$-planes in $T_{o}(\Omega)$. By Theorem 3, gap rigidity in the Zariski topology holds for such pairs $(\Omega, D)$. In fact, we do more: we construct an explicit invariant hypersurface.

(1) Variation of Hodge Structures and Gauss-Manin complexes. Let $\Omega$ be a bounded symmetric domain and $D \subset \Omega$ be a 1-hyperrigid domain in the sense of Eyssidieux [Eys1999] from variation of Hodge structures. The methods and results there apply more generally to period domains. [Eys1999] contains tables of lists of 1-hyperigid subdomains in the case of bounded symmetric domains, but not a full classification. By [Eys1997], Proposition 9.3.6, gap rigidity holds for $(\Omega, D)$ in the Zariski topology, which was proven there by means of the Gauss-Manin complex. There an excluded hypersurface $\mathcal{Z}_{o} \subset \operatorname{Gr}\left(p, T_{o}(\Omega)\right)$ such that $\left[T_{o}(D)\right] \notin \mathcal{Z}_{o}$ can be identified in terms of Lie algebras. Alternatively, for the Gauss-Manin complex $\left(X, K_{p}^{*}\right)$ arising from the variation of Hodge structures, we can find an explicit invariant hypersurface $\mathcal{Z}_{o}$ of $p$-dimensional vector subspaces in $\operatorname{Gr}\left(p, T_{o}(\Omega)\right.$ : this is the locus over which the Gauss-Manin complex fails to be an exact sequence at the point $x \in X$ be described as the zero set at $x$. In other words this the zero set of the determinant of the complex ${ }^{2}$. The condition on scalar curvatures is verified in the calculation there.

(2) Holomorphic curves on certain irreducible bounded symmetric domains. The set of all irreducible bounded symmetric domains $\Omega$ for which there is a $K$-invariant hypersurface is listed in [Mok2002], Proposition 1. For $\operatorname{dim}(D)=1$, gap rigidity holds for $(\Omega, D)$ in the Zariski topology if and only if $\Omega$ belongs to that list and $D$ is a totally-geodesic disk of maximal type, i.e., equivalent under Aut $(\Omega)$ to the diagonal disk of a maximal polydisk (which is of dimension equal to the rank of $\Omega$ ). To put this in the framework of Theorem 3 we list here such domains $\Omega$ together with some invariants.

They will be used again in (4). In the following we normalize the Kähler-Einstein curvature so that the minimal disk is of constant Gaussian curvature -2 . With this normalization the Kähler-Einstein constant $\rho_{\Omega}$ agrees with $-c_{1}(M)$, where $c_{1}(M)$ is the first Chern class of the compact dual $M$, identified in the standard way with a positive integer.

(a) $\Omega$ of Type $I_{m, n}$ with $m=n>1 ; r=n, \operatorname{dim}(\Omega)=n^{2}, \rho_{\Omega}=-2 n$,

(b) $\Omega$ of Type $I I_{n}$ with $n$ even; $r=\frac{n}{2}, \operatorname{dim}(\Omega)=\frac{n(n-1)}{2}, \rho_{\Omega}=-2(n-1)$;

(c) $\Omega$ of Type $I I I_{n}, n \geq 2 ; r=n, \operatorname{dim}(\Omega)=\frac{n(n+1)}{2}, \rho_{\Omega}=-(n+1)$;

(d) $\Omega$ of Type $I V_{n}, n \geq 3 ; r=2, \operatorname{dim}(\Omega)=n, \rho_{\Omega}=-n$; and

(e) $\Omega$ of Type $V I$ (the 27-dimensional exceptional domain pertaining to $E_{7}$ ); $r=3, \operatorname{dim}(\Omega)=27, \rho_{\Omega}=-18$.

Note that the domains $\Omega$ are precisely those of tube type. In retrospect, this can be conceptually explained by the fact that a domain is of tube type iff the diagonal disk embedding is $\left(H_{2}\right)$, [Sat1980], p. 150, Remark 1.

(3) Holomorphic quadric structures. Let $n \geq 3$ and $\Omega=D_{n}^{I V}$ denote the $n$-dimensional bounded symmetric domain of Type IV, which is dual to the $n$ dimensional hyperquadric. In Mok [Mok2002] §4, Theorem 4, we proved that gap

\footnotetext{
${ }^{2}$ This is an invariant holomorphic section on $\operatorname{Gr}\left(p, T_{o} \omega\right)$ of the homogeneous line bundle defined by the determinant of the cohomology, see [GelKapZel1994] for a beautiful exposition of this classical construction of Cayley.
} 
rigidity holds for $\left(D_{n}^{I V}, D_{p}^{I V}, i\right)$ in the Zariski topology for $1 \leq p<n$ and for $i: D_{p}^{I V} \hookrightarrow D_{n}^{I V}$ the standard embedding. For $p=1$ this was included in (2). For $p>1$ we exploited the existence and uniqueness of Kähler-Einstein metrics on projective manifolds with ample canonical line bundles and made use of holomorphic quadric structures. In this case $\left[T_{o}(D)\right] \in \operatorname{Gr}\left(p, T_{o}(\Omega)\right), D=D_{p}^{I V}$ is a semistable point under $K$-action in view of the canonical holomorphic quadric structure $Q$ on $\Omega$. Here at each $x \in \Omega$ we have $Q_{x}: S^{2} T_{x} \rightarrow E_{x}$ where $E$ is a homogeneous holomorphic line bundle on $\Omega$. The excluded subvariety $\mathcal{Z}_{o} \subset \operatorname{Gr}\left(p, T_{o}(\Omega)\right)$ consists precisely of those $p$-planes $V$ such that $\left.Q_{o}\right|_{V}$ is nondegenerate. $\mathcal{Z}_{o}$ is the zero locus of a holomorphic section of a positive power of the dual tautological line bundle on $\operatorname{Gr}\left(p, T_{o}(\Omega)\right)$ corresponding to the discriminant of the (twisted) complex bilinear form $Q_{x}$, which is clearly a hypersurface.

(4) Maximal polydisks on certain irreducible bounded symmetric domains. Let $\Omega$ be one of the irreducible bounded symmetric domains of characteristic codimension 1 as listed in [Mok2002], Proposition 1. Let $r>1$ be its rank. Denote by $\Delta^{r}$ a maximal (totally-geodesic) polydisk in $\Omega$. By (2), gap rigidity holds in the Zariski topology for $\left(\Omega, \delta\left(\Delta^{r}\right)\right)$, where $\delta\left(\Delta^{r}\right)$ stands for the diagonal disk. Actually, gap rigidity also holds for $\left(\Omega, \Delta^{r}\right)$, giving new examples to which Theorem 3 applies. By [Mok2002], Proposition 3, there exists a $G$-invariant holomorphic section $s$ of $L^{-r} \otimes \pi^{*} E$ over $\mathbb{P}\left(T_{\Omega}\right)$, where $L$ stands for the tautological line bundle over $\mathbb{P}\left(T_{\Omega}\right), \pi: \mathbb{P}\left(T_{\Omega}\right) \rightarrow \Omega$ denotes the canonical projection, and $E$ is a homogeneous positive holomorphic line bundle over $\Omega$, such that the zero set of $s$ is precisely the highest characteristic bundle $\pi: \mathcal{S} \rightarrow \Omega$. (Here there is a Zariski open $K^{\mathbb{C}}$ orbit $\mathcal{O}_{o}$ in $\mathbb{P} T_{o}(\Omega)$ and $\mathcal{S}_{o} \subset \mathbb{P}\left(T_{o}(\Omega)\right.$ ) is its complement.) Equivalently $s$ corresponds to a $G$-invariant holomorphic section $\sigma$ of the homogeneous holomorphic vector bundle $S^{r} T_{\Omega}^{*} \otimes E$ on $\Omega$. Writing $T$ for $T_{\Omega}, \sigma$ induces a $K$-invariant linear map $\theta: T \rightarrow S^{r-1} T^{*} \otimes E$, and hence a $K$-invariant map $\wedge^{r} \theta: \Lambda^{r} T \rightarrow \Lambda^{r}\left(S^{r-1} T^{*}\right) \otimes E^{r}$. For a complex vector space $V$ and positive intergers $n$ and $m$ let $\mu: \Lambda^{n}\left(\otimes^{m} V\right) \rightarrow \otimes^{m}\left(\Lambda^{n} V\right)$ be the linear map defined by

$$
\begin{aligned}
& \mu\left(\left(v_{11} \otimes \cdots \otimes v_{1 m}\right) \wedge \cdots \wedge\left(v_{n 1} \otimes \cdots \otimes v_{n m}\right)\right) \\
= & \left(v_{11} \wedge \cdots \wedge v_{n 1}\right) \otimes \cdots \otimes\left(v_{1 m} \wedge \cdots \wedge v_{n m}\right) .
\end{aligned}
$$

If $v_{i j}$ is independent of $j$ then the image under $\mu$ lies in $S^{m}\left(\Lambda^{n} V\right)$. By polarization we conclude that by restriction $\mu: \Lambda^{n}\left(S^{m} V\right) \rightarrow S^{m}\left(\Lambda^{n} V\right)$. Denoting also by $\mu$ the corresponding bundle homomorphism applied to $T$ we obtain a homomorphism $\nu=\left(\mu \otimes i d_{E^{r}}\right) \circ \wedge^{r} \theta, \nu: \Lambda^{r} T \rightarrow S^{r-1}\left(\Lambda^{r} T^{*}\right) \otimes E^{r}$, which therefore defines a $K$ invariant element of $\Lambda^{r} T^{*} \otimes S^{r-1}\left(\Lambda^{r} T^{*}\right) \otimes E^{r}$, and hence by canonical projection a $K$-invariant element $\tau \in S^{r}\left(\Lambda^{r} T^{*}\right) \otimes E^{r}$. Denoting also by $\pi: \mathbb{G}(\Omega) \rightarrow \Omega$ the Grassmann bundle whose fiber over $x \in \Omega$ is the Grassmannian of $r$-planes in $T_{x}(\Omega)$ we have obtained a $G$-invariant holomorphic section $t \in \Gamma\left(\mathbb{G}, L^{-r} \otimes \pi^{*} E^{r}\right)$, where $L$ denotes the tautological line bundle over $\mathbb{G}$.

It remains to show that $t\left(\left[T_{o}\left(\Delta^{r}\right)\right]\right) \notin 0$. Given this, the zero set of $\tau$, which is necessarily non-empty and of codimension 1 in $\mathbb{G}_{o}=\operatorname{Gr}\left(r, T_{o}(\Omega)\right)$, defines the excluded hypersurface $\mathcal{Z}_{o}$, showing that gap rigidity holds in the Zariski topology for $\left(\Omega, \Delta^{r}\right)$. To this end for notational convenience consider the case where $\Omega$ is a Type I domain $D_{r, r}^{I}$ with $r>1$, so that the tangent space can be identified as the space of $r$-by- $r$ matrices. We note that the same argument works in general for any $\Omega$ of characteristic codimension 1 . Denote by $e_{i j}$ the $r$-by- $r$ matrix whose entries are 0 except for the 
$(i, j)$-th entry, which is equal to 1 . Thus $\left(e_{i j}\right)$ constitutes a basis for $T_{o}(\Omega)$, whose dual basis will be denoted by $\left(e_{i j}^{*}\right)$. For the maximal polydisk $\Delta^{r}$ we may take $T_{o}\left(\Delta^{r}\right)$ to consist precisely of the diagonal matrices. Identifying $E_{o}$ with $\mathbb{C}$ the $K$-invariant section $\sigma$ corresponds to the determinant function. Thus $\theta: T \rightarrow S^{r-1} T^{*} \otimes E$ satisfies $\theta\left(e_{i i}\right)=e_{11}^{*} \circ \cdots \circ \widehat{e_{i i}^{*}} \circ \cdots \circ e_{r r}^{*}+$ terms vanishing on $T_{o}\left(\Delta^{r}\right)$, where $\circ$ denotes the symmetric product and $\hat{r}$ denotes exclusion. Hence,

$$
\begin{aligned}
\wedge^{r} \theta\left(e_{11}\right. & \left.\wedge \cdots \wedge e_{r r}\right)=\left(e_{22}^{*} \otimes e_{33}^{*} \otimes \cdots \otimes e_{r r}^{*}\right) \wedge\left(e_{11}^{*} \otimes e_{33}^{*} \otimes \cdots \otimes e_{r r}^{*}\right) \\
\wedge & \cdots
\end{aligned}
$$

It follows by a straightforward calculation that $\nu\left(e_{11} \wedge \cdots \wedge e_{r r}\right)$ is a positive multiple of $\left(e_{11}^{*} \wedge \cdots \wedge e_{r r}^{*}\right)^{r-1}$ modulo terms vanishing on $T_{o}\left(\Delta^{r}\right)$, implying that $\left.t\left(e_{11} \wedge \cdots \wedge e_{r r}\right)^{r}\right) \neq 0$ at $\left.\left[T_{o}\left(\Delta^{r}\right)\right]\right)$, as desired.

If $r=k \ell$ for positive integers $k, \ell>1$, we can write the maximal polydisk as $\Delta^{r}=\left(\Delta^{k}\right)^{\ell}$. Then, the same argument as in the above shows that gap rigidity holds in the Zariski topology for the pair $\left(\Omega,\left(\delta\left(\Delta^{k}\right)^{\ell}\right)\right.$ where $\delta\left(\Delta^{k}\right) \cong \Delta$ is the diagonal.

(5) Examples where the subdomains are higher-dimensional and irreducible. The arguments of (4) give rise to examples of pairs $(\Omega, D)$ with $\Omega=D_{r, r}^{I}$, where $D$ is higher-dimensional and irreducible. These are the subdomains $D=D_{r}^{I I}$, consisting of skew-symmetric matrices, and the subdomains $D=D_{r}^{I I I}$, consisting of symmetric matrices $^{3}$. We have $\operatorname{dim}\left(D_{r}^{I I}\right)=\frac{r(r-1)}{2}$ and $\operatorname{dim}\left(D_{r}^{I I I}\right)=\frac{r(r+1)}{2}$. As in (4) in the process the excluded hypersurface $\mathcal{Z}_{o} \subset \operatorname{Gr}\left(p, T_{o}(\Omega)\right)$ can in principle be explicitly determined. For the purpose of illustration we will establish the semistability of $\left[T_{o}(D)\right]$ in $\operatorname{Gr}\left(p, T_{o}(\Omega)\right.$ in the case of $r=3$.

We start with $D=D_{3}^{I I I}$, which is a 6 -dimensional bounded symmetric domain of rank 2, Let $\left(e_{i j}\right)$ be a basis of $T_{o}(\Omega)$ and $\left(e_{i j}^{*}\right)$ be a dual basis, as in (4). Then, $T_{o}(D)$ is spanned by the basis $\left\{e_{12}+e_{21}, e_{13}+e_{31}, e_{23}+e_{32}, e_{11}, e_{22}, e_{33}\right\}$. We write $x_{i j}$ for $e_{i j} \bmod T_{o}(D)$. For the determinant det on 3 -by-3 matrices, we have

$$
\operatorname{det}=e_{11}^{*} e_{22}^{*} e_{33}^{*}+e_{12}^{*} e_{23}^{*} e_{31}^{*}+e_{13}^{*} e_{21}^{*} e_{32}^{*}-e_{13}^{*} e_{22}^{*} e_{31}^{*}-e_{11}^{*} e_{23}^{*} e_{32}^{*}-e_{12}^{*} e_{21}^{*} e_{33}^{*},
$$

so that

$$
\theta\left(e_{12}\right)=e_{23}^{*} e_{31}^{*}-e_{21}^{*} e_{33}^{*}, \quad \theta\left(e_{21}\right)=e_{13}^{*} e_{32}^{*}-e_{12}^{*} e_{33}^{*} .
$$

Denote by $\bar{\theta}$ the composite $\kappa \circ \theta$, where $\kappa$ is the projection map induced by the quotient homomorphism $T_{o}^{*}(\Omega) \rightarrow T_{o}^{*}(D)$. We have

$$
\bar{\theta}\left(e_{12}\right)=x_{23} x_{31}-x_{21} x_{33}, \quad \bar{\theta}\left(e_{21}\right)=x_{13} x_{32}-x_{12} x_{33} .
$$

Noting that $x_{i j}=x_{j i}$ we have

$$
\bar{\theta}\left(e_{12}\right)=x_{23} x_{13}-x_{12} x_{33}=\bar{\theta}\left(e_{21}\right),
$$

implying by analogous calculations

\footnotetext{
${ }^{3}$ In some cases, $D \subset \Omega$ occurred as a 1-hyperrigid subdomain, namely $D_{r}^{I I}$ and $D_{r}^{I I I}, r \cong 0,1[4]$, see [Eys1999].
} 


$$
\begin{aligned}
& \bar{\theta}\left(e_{12}+e_{21}\right)=2\left(x_{23} x_{13}-x_{12} x_{33}\right) ; \\
& \bar{\theta}\left(e_{13}+e_{31}\right)=2\left(x_{12} x_{23}-x_{13} x_{22}\right) ; \\
& \bar{\theta}\left(e_{23}+e_{32}\right)=2\left(x_{12} x_{13}-x_{23} x_{11}\right)
\end{aligned}
$$

On the other hand

$$
\theta\left(e_{11}\right)=e_{22}^{*} e_{33}^{*}-e_{23}^{*} e_{32}^{*},
$$

implying by analogous calculations

$$
\bar{\theta}\left(e_{11}\right)=x_{22} x_{33}-x_{23}^{2}, \quad \bar{\theta}\left(e_{22}\right)=x_{11} x_{33}-x_{13}^{2}, \quad \bar{\theta}\left(e_{33}\right)=x_{11} x_{22}-x_{12}^{2} .
$$

Putting together the formulae for $\bar{\theta}$ for the basis vectors $\left\{e_{12}+e_{21}, e_{13}+e_{31}\right.$, $\left.e_{23}+e_{32}, e_{11}, e_{22}, e_{33}\right\}$, we obtain a formula for the section $t \in \Gamma\left(\mathbb{G}_{o}, L^{-3}\right)$. Denoting by $\bar{\mu}$ the homomorphism induced by $\mu: \Lambda^{6}\left(S^{2} T_{o}\right) \rightarrow S^{2}\left(\Lambda^{6} T_{o}\right)$ as in (4), and by the quotient homomorphism $T_{o}^{*} \rightarrow T_{o}^{*}(D)$, we have

$$
\begin{aligned}
& t\left(\left(e_{12}+e_{21}\right) \wedge\left(e_{13}+e_{31}\right) \wedge\left(e_{23}+e_{32}\right) \wedge e_{11} \wedge e_{22} \wedge e_{33}\right) \\
& =8 \bar{\mu}\left(\left(x_{23} x_{13}-x_{12} x_{33}\right) \wedge\left(x_{12} x_{23}-x_{13} x_{22}\right) \wedge\left(x_{12} x_{13}-x_{23} x_{11}\right)\right. \\
& \left.\wedge\left(x_{22} x_{33}-x_{23}^{2}\right) \wedge\left(x_{11} x_{33}-x_{13}^{2}\right) \wedge\left(x_{11} x_{22}-x_{12}^{2}\right)\right) .
\end{aligned}
$$

The only terms that count come from the combination of the first halves of each of the 6 quadratic expressions inside brackets, since all other exterior products are zero, as the variables $\left(x_{23}, x_{12}, x_{13}, x_{11}, x_{22}, x_{33}\right)$ do not occur the right number of times. We get finally

$$
\begin{aligned}
& t\left(\left(e_{12}+e_{21}\right) \wedge\left(e_{13}+e_{31}\right) \wedge\left(e_{23}+e_{32}\right) \wedge e_{11} \wedge e_{22} \wedge e_{33}\right) \\
& \quad=32\left(x_{23} \wedge x_{12} \wedge x_{13} \wedge x_{22} \wedge x_{33} \wedge x_{11}\right)^{2} \neq 0,
\end{aligned}
$$

as desired. The zero set of $t$ defines the cubic hypersurface $\mathcal{Z}_{o} \subset \mathbb{G}_{o}$ on the Grassmannian $\mathbb{G}_{o}$ which serves as the excluded subvariety in Theorem 3.

Next we consider $D=D_{3}^{I I}$, which is a 3 -dimensional bounded symmetric domain of rank 1, i.e., biholomorphic to the 3-dimensional unit ball $B^{3}$. A straightforward adaptation of the preceding argument does not work. Use the same notations as in the above, except that $D$ stands for $D_{3}^{I I}$ and that $x_{i j}$ is $e_{i j}^{*}$ modulo $T_{o}(D)$, i.e., the vector subspace of skew-symmetric matrices in the vector space of 3-by-3 matrices, so that $x_{i j}=-x_{j i}$, in particular $x_{33}=0$, and we have

$$
\begin{array}{r}
\bar{\theta}\left(e_{12}\right)=x_{23} x_{31}, \bar{\theta}\left(e_{21}\right)=x_{13} x_{32} \\
\bar{\theta}\left(e_{21}\right)=\left(-x_{31}\right)\left(-x_{23}\right)=x_{23} x_{31}=\bar{\theta}\left(e_{12}\right), \quad \text { hence } \\
\bar{\theta}\left(e_{12}-e_{21}\right)=0 .
\end{array}
$$

It follows that we get the section $t \equiv 0$ in this case. To overcome the difficulty, we work with symmetric matrices in place of skew-symmetric matrices by going to the 
cotangent bundle, as follows. The starting point of the construction of $\mathcal{Z}$ is the existence of the determinant. On the compact dual $M$, which is here the Grassmannian $\operatorname{Gr}(r, V)$ of 3-planes on $V \cong \mathbb{C}^{6}$, and at a point $x \in M$, identifying $T_{x}(M)$ with the set of 3-by-3 matrices, we can define tentatively the 'determinant' of the tangent vector. However, the matrix reprentation is unique only up to the complexification $K^{\mathbb{C}}$ of the isotropy subgroup $K$ at $o$. The action of the centre of $K_{x}$ on the 'determinant' shows that there is a well-defined determinant on the projectivized tangent bundle of $M$, as an $\operatorname{Aut}(M)$-invariant section of a homogenenous holomorphic line bundle. Likewise we can define a determinant for the cotangent bundle as a section on $\mathbb{P}\left(T^{*}(M)\right)$, with tautological line bundle $\Lambda$, of a homogeneous holomorphic line bundle which restricts to $\Lambda^{-r}$ over $\mathbb{P}\left(T_{o}^{*}(M)\right)$, corresponding to an element of $S^{r} T_{o}(M)$. Now in general there is a canonical correspondence between the Grassmann bundle $\mathbb{G}(M)$ over $M$ of $p$-dimensional tangent planes over $M$ with the Grassmann bundle $\mathbb{G}^{\prime}$ of $(m-p)$ dimensional vector subspaces of cotangent spaces over $M$, obtained by sending each $p$-plane $A \subset T_{x}(M)$ to its annihilator $A^{\perp} \subset T_{x}^{*}$. Consider a reference point $o \in \Omega$ and represent $T_{o}(\Omega)$ as the space of 3-by-3 matrices such that $T_{o}(D)$ is identified with the 3 -dimensional vector subspace of skew-symmetric matrices. Identifying also $T_{o}^{*}(\Omega)$, via the natural complex bilinear pairing between $T_{o}(\Omega)$ and $T_{o}^{*}(\Omega)$, as the vector space of 3-by-3 matrices. Then $\left(T_{o}(D)\right)^{\perp}$ is nothing other than the 6 -dimensional vector space of symmetric matrices. Working with the determinant on the cotangent bundle in place of the tangent bundle we can perform the same proof as in the above to find a cubic hypersurface in $\mathbb{G}_{o}^{\prime}$ which avoids the point $\left[T_{o}^{\perp}(D)\right]$. The canonical isomorphism $\mathbb{G}_{o}(D) \cong \mathbb{G}_{o}^{\prime}(D)$ then gives a cubic hypersurface $\mathcal{Z}_{o} \subset \mathbb{G}_{o}^{\prime}$ which avoids $\left[T_{o}(D)\right]$.

Remarks. (a) Examples discuss in (2) - (5) are all obtained on irreducible bounded symmetric domains $\Omega$ of characteristic codimension 1, i.e., those admitting a $K$ invariant hypersurface. This does not need to be the case for $(\Omega, D)$ to exhibit gap rigidity for some choice of $D$. Such examples occur in the context of Gauss-Manin complexes. For certain values of $p, n>1$, and for $\Omega=D_{n, p n}^{I}$ it was shown in Eyssidieux [Eys1999] that $\left(D_{n, p n}^{I}, B^{p} ; i\right)$ is 1-hyperrigid, where $i: B^{p} \rightarrow D_{n, p n}^{I}$ is obtained by embedding $B^{p}$ as the diagonal of a product $P=B^{p} \times \cdots \times B^{p}$ ( $n$ factors) of complex unit $p$-balls, and $P$ is realized as a totally geodesic submanifold of $\Omega$ in a standard way. This was precisely the hint towards the general theory.

(b) The method used in (4) and (5) can be applied to yield various examples where $(\Omega, D)$ exhibits gap rigidity in the Zariski topology. They are typically of 'diagonal' type, for instance, if $n=p \ell$, then $\left(D_{n}^{I I I}, \delta\left(\left(D_{p}^{I I I}\right)^{\ell}\right)\right)$ can be shown to exhibit gap rigidity in the Zariski topology. The methods of (4) and (5) produce in each case a locally homogeneous holomorphic section $t$ in some $\Gamma\left(\mathbb{P}\left(T_{X}\right), L^{-m} \otimes \pi^{*} E\right)$, and the question is to know whether $t\left(\left[T_{o}(D)\right]\right) \neq 0$. As is shown in (5), it can happen that $t \equiv 0$, although in that particular example there is a way to circumvent the difficulty. When however $t \not \equiv 0$, then there is the advantage that $t$ can in principle be explicitly determined. Moreover, by [Mok2002], Proposition 3, whenever the higher characteristic subvariety $\mathcal{S}_{o} \subset \mathbb{P} T_{o}(\Omega)$ is a hypersurface, it is always of degree $r=$ $\operatorname{rank}(\Omega)$. When $t \neq 0$, the construction in (4) and (5) yields a symmetric polynomial of degree $r$. Thus, in the case of (3) on holomorphic quadric structures, the excluded hypersurface $\mathcal{Z}_{o} \subset \mathbb{G}_{o}$ is always the zero set of a nontrivial quadratic polynomial.

2.6. Classification of $\left(H_{3}\right)$-embeddings into an irreducible domain. An embedding into a reducible domain is $\left(H_{2}\right)$ if and only if its factors are also $\left(H_{2}\right)$ embeddings. This reduces the classification of $\left(H_{2}\right)$-embeddings to the classification of maximal $\left(H_{2}\right)$-embeddings into an irreducible domain. We give two tables, ex- 
tracted from [Iha1967] and [Sat1980]. The first one gives all maximal $\left(H_{2}\right)$-embeddings into classical domains up to equivalence. The second table gives all maximal $\left(H_{2}\right)$ embeddings into an exceptional domains and, for every irreducible $\left(H_{2}\right)$-subdomain, a chain relating it to a maximal one.

$\left(H_{3}\right)$-embeddings $(\Omega, D)$ into an irreducible domain $\Omega$ are $\left(H_{2}\right)$ embeddings with an additional requirement on the Einstein constants of the factors of $D$ if $D$ is not irreducible. It is straightforward to use the tables to give a complete list of all $\left(H_{2}\right)$ embeddings into a given irreducible domain, and it is easy in each case to compute the Einstein constant of the induced metric on every irreducible component and decide whether they agree, giving a complete list of $\left(H_{3}\right)$-embeddings into $\Omega$. We will be content with the remark that a way to check without calculation that a $\left(H_{2}\right)$ embedding of a reducible domain of the form $D=\Omega_{1}^{n}$, where $\Omega_{1}$ is irreducible, is $\left(H_{3}\right)$ when the automorphism group of $\Omega$ permutes the various subdomains of the form $o \times \ldots \times o \times \Omega_{1} \times o \times \ldots$ This gives $\left(H_{3}\right)$-embeddings of the form $\left(D_{k p, k q}^{I},\left(D_{p, q}^{I}\right)^{k}\right)$, $\left(D_{n k}^{A},\left(D_{n}^{A}\right)^{k}\right) A=I I, I I I,\left(D_{20 p}^{I I I},\left(B^{5}\right)^{p}\right)$, etc. In the following tables of maximal $\left(H_{2}\right)$-subdomains, in the case where $D$ is irreducible, the $\left(H_{3}\right)$-condition is satisfied precisely for $D$ of this form. $\left(H_{3}\right)$-embeddings into a reducible domain are also in principle straightforward to classify.

\begin{tabular}{|c|c|c|c|}
\hline \multicolumn{5}{|c|}{$\begin{array}{c}\text { Maximal }\left(H_{2}\right) \text {-subdomains } \\
\text { of a classical domain }\end{array}$} \\
\hline$\Omega$ & $D$ & maximal & Additional conditions \\
\hline$D_{p, q}^{I}$ & $D_{r, s}^{I} \times D_{p-r, q-s}^{I}$ & $*$ & $\begin{array}{c}r \\
s\end{array}=\frac{p}{q}$ \\
& & & $\left(H_{3}\right)$ iff $p=2 r$ \\
\hline & $D_{n}^{I I}$ & $*$ & $p=q=n$ \\
\hline & $D_{n}^{I I}$ & $*$ & $p=q=n$ \\
\hline & $B^{m}$ & $m \neq 2 r+1$ & $p=\left(\begin{array}{c}m \\
r-1\end{array}\right), q=\left(\begin{array}{c}m \\
r\end{array}\right), r \in \mathbb{N}$ \\
\hline & $D_{2 l}^{I V}$ & $l \equiv 0[2]$ & $p=q=2^{l}, l \geq 3$ \\
\hline & $D_{2 l-1}^{I l}$ & & $p=q=2^{l-1}, l \geq 3$ \\
\hline$D_{n}^{I I}$ & $D_{r, r}^{I}$ & $*$ & $n=2 r$ \\
\hline & $D_{r}^{I I} \times D_{n-r}^{I I}$ & $*$ & $n>r$ \\
& & & $\left(H_{3}\right)$ iff $n=2 r$ \\
\hline & $B^{m}$ & $*$ & $n=\left(\begin{array}{c}m+1 \\
\frac{m+1}{2}\end{array}\right), m \equiv 3[4]$ \\
\hline & $D_{2 l}^{I V}$ & $*$ & $n=2^{l}, l \geq 3, l \equiv 3[4]$ \\
\hline & $D_{2 l-1}^{I V}$ & $*$ & $n=2^{l-1}, l \geq 3, l \equiv 0,3[4]$ \\
\hline$D_{n}^{I I I}$ & $D_{r, r}^{I}$ & $*$ & $n=2 r$ \\
\hline & $D_{r}^{I I} \times D_{n-r}^{I I I}$ & $*$ & $n>r$ \\
& & $*$ & $\left(H_{3}\right)$ iff $n=2 r$ \\
\hline & $B^{m}$ & $*$ & $n=\left(\begin{array}{c}m+1 \\
\frac{m+1}{2}\end{array}\right), m \equiv 1[4]$ \\
\hline & $D_{2 l}^{I V}$ & $*$ & $p=q=2^{l}, l \geq 3, l \equiv 1[4]$ \\
\hline & $D_{2 l-1}^{I V}$ & $*$ & $p=q=2^{l-1}, l \geq 3, l \equiv 1,2[4]$ \\
\hline$D_{2 l}^{I V}$ & $D_{2,2}^{I}$ & & $l \geq 3$ \\
\hline & $D_{2 l-1}^{I V}$ & $*$ & $l \geq 3$ \\
\hline$D_{2 l-1}^{I V}$ & $D_{2 l-2}^{I V}$ & $*$ & $l \geq 3$ \\
\hline & & & $*$ \\
\hline
\end{tabular}




\begin{tabular}{|c|c|c|c|}
\hline \multicolumn{5}{|c|}{ Maximal and irreducible $\left(H_{2}\right)$-subdomains } \\
of exceptional domains
\end{tabular}

\section{Overview on gap rigidity.}

3.1. Gap rigidity for $D_{2}^{I I I}$. From [Mok2002], Theorem 4, (or Theorem 3 here) and Theorem 1, we have a complete understanding of gap rigidity for the unique 3 -dimensional bounded symmetric domain $\Omega$ of rank $>1$. $\Omega$ can be described as a Type III domain of rank 2, equivalently the 3-dimensional Siegel upper half-plane, or as the 3-dimensional bounded symmetric domain of Type IV, i.e., the noncompact dual of the 3-dimensional hyperquadric.

Proposition 3. The question on the validity of gap rigidity on the 3-dimensional irreducible bounded symmetric domain $\Omega$ of rank $>1$ is completely settled, as follows. Denote by $\Delta^{2} \subset \Omega$ a maximal totally-geodesic bidisk as given by the Polydisk Theorem. There are, up to isometry, precisely 3 different types of positive-dimensional totallygeodesic proper complex submanifolds $D$ of $\Omega$, namely:

(1) $D=\Delta^{2} \subset \Omega$;

(2) $D=\delta(\Delta) \subset \Delta^{2} \subset \Omega$;

(3) $D=\Delta \times\{0\} \subset \Delta^{2} \subset \Omega$.

Gap rigidity holds in the Zariski sense for $(\Omega, D)$ for $D$ in (1) or (2); but fails (in the complex topology) for $D$ in (3).

3.2. Rank one domains. Theorem 3 and the examples in (2.5) show that gap rigidity in the Zariski topology for a pair $(\Omega, D)$ can hold due to algebraic conditions satisfied by tangent planes to bounded symmetric domains. Theorem 1 shows that gap rigidity can fail in the complex topology due to product structures, although the construction of counterexamples arising from holomorphic maps between compact Riemann surfaces of higher genus does not generalize easily (cf. (3.3)). There is a situation which belongs to neither of these situations and for which gap rigidity in the Zariski topology does not make sense. This is especially the case for bounded symmetric domains of rank 1.

QUESTION 1. Let $k<n$ be positive integers and embed the complex unit $k$-ball $B^{k}$ into the complex unit $n$-ball $B^{n}$ in the standard way as a totally geodesic complex submanifold. Does gap rigidity hold for $\left(B^{n}, B^{k}\right)$ in the complex topology?

There is up to this point no evidence as to whether one should expect a positive or negative answer to Question 1. Since the information of first order is trivial in this problem, perhaps one should try and find a way to deal with higher order information, which we cannot do for the time being. The case of $k=1$ is perhaps the most difficult. A negative answer for $k=1, n=2$ constructed on quotients of the two-ball by torsion-free lattices would give first examples of exceptional divisors other than 
totally-geodesic cycles on some projective manifolds uniformized by the 2-ball. The case of $k>1$ can be formulated perhaps as a problem in two steps. The first step is to understand whether a sufficiently pinched $k$-dimensional submanifold $S$ with $k>1$ is necessarily uniformized by $B^{k}$. This is equivalently the question of asking whether $S$ admits a holomorphic projective structure, in view of [KobOch1981]. In the case of $k=2$ it was conjectured in Siu-Yang [SiuYan1981] that a compact Kähler-Einstein surface with strictly negative sectional curvature is uniformized by the complex unit 2-ball $B^{2}$. In the same article, they proved a type of pinching theorem which says that a compact Kähler-Einstein surface of nonpositive holomorphic bisectional curvature must be biholomorphic to the 2-ball, provided that the curvature at every point satisfies some very specific pinching condition. It is possible to deform an $\epsilon$-pinched compact complex submanifold $S \subset X:=B^{n} / \Gamma$ to get a Kähler-Einstein surface with estimates on the deviation from constant holomorphic sectional curvature. Unfortunately the pinching condition in [SiuYan1981] is not implied by such estimates. The second step of the problem is to prove that a holomorphic immersion of $S=B^{k} / \Gamma_{o}$ into $X=B^{n} / \Gamma$ is necessarily a totally geodesic immersion, at least when the image is sufficiently pinched. In the case where $2 k>n$ this was settled in the positive in Cao-Mok [CaoMok1990] without assuming any pinching condition. In particular, in the case where $k=2, n=3$ Question 1 is reduced to the first step.

3.3. Embeddings into products. In relation to the counterexamples showing that gap rigidity does not hold for $\left(\Delta^{2}, \Delta \times o\right)$, one can ask the question as to whether this type of construction can generalize. Obviously one can get holomorphic mappings between products of compact Riemann surfaces of genus $>2$ to show that gap rigidity fails for $\left(\Omega, \Delta^{k} ; i\right)$ with $\operatorname{rank}(\Omega)=r>1, \Omega$ not necessarily irreducible, $k<r$ and $i: \Delta \rightarrow \Omega$ arising from the inclusion $\Delta^{k}=\Delta^{k} \times\{0\} \subset \Delta^{r}$, and an embedding of $\Delta^{r}$ as a maximal polydisk in $\Omega$. On the other hand, for $\Omega$ an irreducible bounded symmetric domain of rank $>1$, we have

Proposition 4 . Let $\Omega$ be an irreducible bounded symmetric domain of rank $>1$. Let $k<n$ be positive integers. Then, gap rigidity holds for the pair $\left(\Omega^{n}, \delta\left(\Omega^{k}\right) \times\{0\}\right)$ in the Zariski topology when we consider only ambient complex manifolds $X$ of the form $\Omega^{n} / \Gamma$ with $\Gamma \subset$ Aut $_{o}\left(\Omega^{n}\right)$.

Proof. Write $\Omega=G / K$ in the usual notations. Here we can define, in the notations analogous to the statement of Theorem $3, \mathcal{Z}_{o} \subset \mathbb{G}_{o}$ to consist of tangent $p$ planes $V, p=\operatorname{dim}(\Omega)$, such that the canonical projection of $V$ onto, say the first factor $\Omega$ is an isomorphism. With this convention, given any $S \subset X / \Gamma$, where $\Gamma \subset A u t_{o}\left(\Omega^{n}\right)$, the canonical projection $\rho: \Omega^{n} \rightarrow \Omega$ onto the first factor induces on $S$ an integrable $K^{\mathbb{C}}$-structure. It follows that the universal covering space of $S$ is biholomorphic to $\Omega$ (cf. [MokYeu1992]). Thus, we have $S=\Omega / \Gamma_{o}$ as an abstract complex manifold, together with a holomorphic embedding $f: S \rightarrow X$. By Hermitian metric rigidity of Mok [Mok1987] it follows that $f: S \rightarrow X$ is a totally geodesic isometric embedding up to a nonzero normalizing constant. Identifying $S$ with its image under $f$ we conclude that $S \subset X$ is a holomorphic geodesic cycle.

Proposition 4 gives an example where gap rigidity can hold in the Zariski topology, with a reducible ambient domain $\Omega^{n}$, even though the analogous condition on scalar curvatures as in Theorem 3 is not satisfied. Here of course we are dealing with reducible ambient domains, otherwise even the notion of gap rigidity in the Zariski topology as stated cannot be formulated, but one may still raise the question, in the 
case of a pair $(\Omega, D)$ with $\Omega$ an irreducible bounded symmetric domain, whether gap rigidity in the complex topology can hold for certain bounded symmetric domains $D$ which are say irreducible and of rank $\geq 2$. The problem can be reduced to a question of holomorphic G-structures and is not within the scope of methods in the current article.

Finally, it is intriguing even in the reducible case, whether one can construct counterexamples to gap rigidity for pairs $\left(B^{n} \times B^{n}, \delta\left(B^{n} \times B^{n}\right)\right)$ with $n>1$. We may formulate the problem as follows

QUestion 2. Let $n>1$. Consider the set $\mathcal{X}_{n}$ of all compact complex manifolds uniformized by the complex unit ball $B^{n}$. Let $\operatorname{Map}\left(\mathcal{X}_{n}\right)$ denote the set of all nonconstant holomorphic mappings $f: X \rightarrow X^{\prime}$ with $X, X^{\prime} \in \mathcal{X}_{n}$, and $\operatorname{Map}_{f i n}\left(\mathcal{X}_{n}\right) \subset \operatorname{Map}\left(\mathcal{X}_{n}\right)$ the subset of all generically finite holomorphic maps. For each $f \in \operatorname{Map}\left(\mathcal{X}_{n}\right), f: X \rightarrow X^{\prime}$, denote by $\mu(f) \in(0,1]$ the real number defined by $\mu(f)=\sup \{\|d f(x)\|: x \in X\}$. Does there exist a universal constant $c_{n}>0$ depending only on $n$ such that $\mu(f)>c_{n}$ for any $f \in \operatorname{Map}_{f i n}\left(\mathcal{X}_{n}\right)$ or more generally for $f \in \operatorname{Map}\left(\mathcal{X}_{n}\right)$ ?

We note that $\mu(f) \leq 1$ because of the Ahlfors-Schwarz Lemma. A negative answer to Question 2 for a given $n>1$ will imply the failure of gap rigidity in the complex topology for $\left(B^{n} \times B^{n}, B^{n} \times\{0\}\right)$. On the other hand, a positive answer to an extended form of the question, where in place of $f \in \operatorname{Map}\left(\mathcal{X}_{n}\right)$ we consider compact complex manifolds $X \in \mathcal{X}_{n}$, any representation $\Phi: \pi_{1}(X) \rightarrow \operatorname{Aut}\left(B^{n}\right)$ and any $\Phi$-equivariant holomorphic map $F: B^{n} \rightarrow B^{n}$, will lead to the confirmation of the gap rigidity in the complex topology for the pair $\left(B^{n} \times B^{n}, B^{n} \times\{0\}\right)$.

\section{REFERENCES}

[Bor1963]

[CaoMok1990]

[Eys1997]

[Eys1999]

[EysMok1995]

[GelKapZel1994]

[Iha1966]

[Iha1967]

[Kir1984]

[KobOch1981]

[MarWei1974]
Borel, A., Compact Clifford-Klein forms of symmetric spaces, Topology, 2 (1963), pp. 111-122.

CaO, H.-D. AND MoK, N., Holomorphic immersions between complex hyperbolic space forms, Invent. Math., 100 (1990), pp. 49-61.

Eyssidieux, P., La caractéristique d'Euler du complexe de Gauss-Manin, J. reine angew. Math., 490 (1997), pp. 155-212.

Eyssidieux, P., Kähler hyperbolicity and variations of Hodge structures, in New Trends in Algebraic Geometry, ed. K. Hulek, F. Catanese, C. Peters and M. Reid, Cambridge University Press (1999), pp. 71-92.

Eyssidieux, P. AND Mok, N., Characterization of certain holomorphic geodesic cycles on Hermitian locally symmetric manifolds of the noncompact type, in Modern Methods in Complex Analysis: The Princeton Conference in Honor of Gunning and Kohn, ed. T. Bloom, D. Catlin, J. D'Angelo and Y.T. Siu; Annals of Mathematics Studies, Volume 138, Princeton University Press (1995), pp. 85-118.

Gel'fand, I., Kapranov, M., And Zelevinsky, A., Discriminants, resultants, and multidimensional determinants, Mathematics: Theory and Applications, Birkhauser Boston, (1994).

IHARA, S., Holomorphic imbeddings of symmetric domains into a symmetric domain, Proc. Japan Acad. Sci., 42 (1966), pp. 193-197.

IharA, S., Holomorphic imbeddings of symmetric domains, J. Math. Soc. Japan, 19, pp. 261-302; Supplement, ibid., pp. 543-544.

KiRwan, F., Cohomology of quotients in symplectic and algebraic geometry, Math. Notes, 31, Princeton University Press (1984).

Kobayashi, S. And Ochiai, T., Holomorphic projective structures on compact complex surfaces, Math. Ann., 249 (1980), pp. 75-94.

Marsden, J. And Weinstein, A., Reduction of symplectic manifolds with symmetry, Rep. Math. Phys., 5, 121 (1974). 
[Mok1987] MoK, N., Uniqueness theorems of Hermitian metrics of seminegative curvature on locally symmetric spaces of negative Ricci curvature, Ann. Math., 125 (1987), pp. 105-152.

[Mok2002] Mok, N., Characterization of certain holomorphic geodesic cycles on quotients of bounded symmetric domains in terms of tangent subspaces, Comp. Math., 132 (2002), pp. 289-309.

[MokYeu1992] MoK, N. AND YEUnG, S.-K., Geometric realization of uniformization of Hermitian locally symmetric manifolds, in Complex Analysis and Geometry, ed. by Ancona and Silva, New York, London (1992), pp. 253-270.

[MumFogKir1994] Mumford, D., Fogarty, J. and Kirwan, F., Geometric Invariant Theory, 3rd edition, Erg. der Math. 3. Folge 34, Springer (1994).

[Sat1965]

[Sat1980]

[SiuYan1981]

[Sou1970]

[Wol1972]

Satake, I., Holomorphic imbeddings of symmetric domains into a Siegel space, Amer. J. Math., 90 (1965), pp. 425-461.

Satake, I., Algebraic structures of symmetric domains, Publications of the Mathematical Society of Japan 14, Iwanami Shoten and Princeton University Press (1980).

Y.T. SiU AND P. YANG, Compact Kähler-Einstein surface of nonpositive bisectional curvature, Invent. Math., 64:3 (1981), pp. 471-487.

Souriau, J., Structures des systèmes dynamiques, Dunod (1970).

Wolf, J.A., Fine structure of Hermitian symmetric spaces, In: W. Boothby and G. Weiss (eds), Geometry of Symmetric Spaces, Marcel Dekker (1972), pp.271-357. 